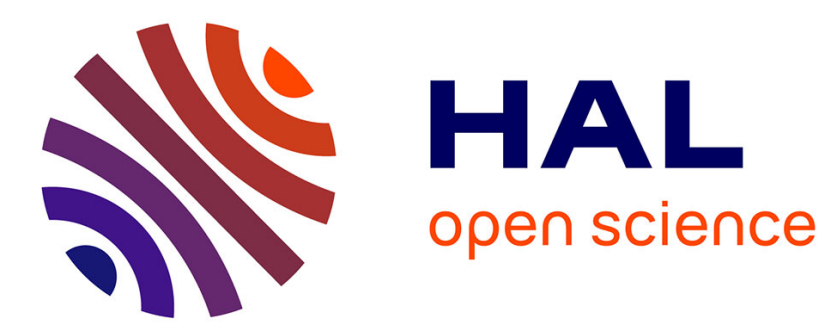

\title{
Axisymmetric eddy current inspection of highly conducting thin layers via asymptotic models
}

Houssem Haddar, Zixian Jiang

\section{To cite this version:}

Houssem Haddar, Zixian Jiang. Axisymmetric eddy current inspection of highly conducting thin layers via asymptotic models. Inverse Problems, 2015. hal-01214308

\author{
HAL Id: hal-01214308 \\ https://hal.inria.fr/hal-01214308
}

Submitted on 11 Oct 2015

HAL is a multi-disciplinary open access archive for the deposit and dissemination of scientific research documents, whether they are published or not. The documents may come from teaching and research institutions in France or abroad, or from public or private research centers.
L'archive ouverte pluridisciplinaire HAL, est destinée au dépôt et à la diffusion de documents scientifiques de niveau recherche, publiés ou non, émanant des établissements d'enseignement et de recherche français ou étrangers, des laboratoires publics ou privés. 


\title{
Axisymmetric eddy current inspection of highly conducting thin layers via asymptotic models
}

\author{
Houssem Haddar*, Zixian Jiang ${ }^{\dagger}$
}

\begin{abstract}
Thin copper deposits covering the steam generator tubes can blind eddy current probes in nondestructive testings of problematic faults and are therefore important to be identified. Existing methods based on shape reconstruction using eddy current signals encounter difficulties of high numerical costs due to the layer's small thickness and high conductivity. In this article, we approximate the axisymmetric eddy current problem with some appropriate asymptotic models using effective transmission conditions representing the thin deposits. In these models, the geometrical information related to the deposit is transformed into parameter coefficients on a fictitious interface. Standard iterative inversion algorithm is then applied to the asymptotic models to reconstruct the thickness of the thin copper layers. Numerical tests both validating the asymptotic model and showing benefit of the inversion procedure are provided. Keywords: axisymmetric eddy current inspection, asymptotic model, thickness reconstruction of thin layers.
\end{abstract}

\section{Introduction}

Thin layers of copper deposits are observed in non-destructive eddy current testing of steam generator tubes for the safety and failure-free operating of nuclear power plants. Covering the shell side of the tube, these deposits lead to a significant signal feedback in the eddy current inspection due to the high conductivity, despite their extremely small thickness (see Table 1 for a comparison with the tube). These deposits do not directly effect the productivity of steam nor the safety of structure. However their presence may mask other kinds of problematic faults such as clogging magnetite deposits, cracks in tubes, etc. This is why it is important to identify them.

\begin{tabular}{lll}
\hline & tube wall & copper layer \\
\hline conductivity (in $\left.S \cdot m^{-1}\right)$ & $\sigma_{t}=0.97 \times 10^{6}$ & $\sigma_{c}=58.0 \times 10^{6}$ \\
thickness (in $m m$ ) & $r_{t_{2}}-r_{t_{1}}=1.27$ & $0 \leq f_{\delta}(z) \leq 0.1$ \\
\hline
\end{tabular}

Table 1: Conductivity and scale differences between tube wall and copper layer.

In previous studies, we modeled the axisymmetric eddy current problem with an efficient finite element approximation which involves artificial boundary conditions to cut off the computational domain and thus reduce the numerical cost (see [10]). Based on this model is developed an inversion algorithm using shape optimization methods to reconstruct the shape of some clogging magnetite deposits [12]. These methods can be naturally applied to thickness reconstruction of thin copper deposits. However, a major numerical challenge to deal with this case is the high numerical cost resulting from the fact that the computational domain should be discretized into a fine mesh with the same scale to the layer thickness of the copper deposits.

Besides adaptive mesh refining method (see for example [7]), another widely practiced strategy to reduce computational cost consists in modeling the thin deposit with effective transmission conditions (see for example [19], 21]). [20] in particular supplies analysis and comparison of different impedance transmission conditions for thin sheets in two dimensional eddy current model. These transmission conditions are obtained by matching the asymptotic expansions of the solution with respect to a small parameter characterizing the

*houssem.haddar@inria.fr, DÉFI team, INRIA Saclay, Palaiseau, France.

${ }^{\dagger}$ zixian.jiang@polytechnique.edu, DÉFI team, INRIA Saclay, Palaiseau, France. 
thickness of the thin deposit, hence the use of the term asymptotic model. From the inverse problem perspective, an additional major advantage of asymptotic model is to avoid re-meshing at each iteration. A rich literature on asymptotic models has been developed for different approaches and various applications (see for example [3], [2], [6], 5], 4], [18], [13, [14, [22] and the references therein).

In the research note [9] the authors built and compared several asymptotic models using a family of effective transmission conditions $\left\{\mathcal{Z}_{m, n}\right\}_{m, n \in\{0,1,2\}}$ for the axisymmetric eddy current inspection of thin copper deposits. Roughly speaking, if the thickness of a thin deposit at vertical position $z$ of the tube writes $f_{\delta}(z)=\delta d(z)$, then the index $m$ of the transmission condition $\mathcal{Z}_{m, n}$ denotes a re-scaling parameter of the copper conductivity with regard to $\delta: \sigma_{c}=\sigma_{m} /\left(\delta^{m}\right)$, while the index $n$ stands for the order of asymptotic expansion of the solution with respect to $\delta$. Numerical tests therein on a reduced 1D case with constant deposit thickness show that the asymptotic models with $\mathcal{Z}_{1, n}(n=0,1)$ conditions give satisfying approximation of the full model and lead to easy construction of inversion methods.

In this article we first introduce the axisymmetric model for eddy current testing and some useful techniques to develop the asymptotic models (Section 2). Using the same method presented in [9], we then build the variational asymptotic models with $\mathcal{Z}_{1, n}(n=0,1)$ transmission conditions for general cases with variable deposit thickness (Section 3). By introducing adjoint states associated to derivatives of the impedance measurements, we formulate the inverse problem for thickness reconstruction as the minimization of a least square cost functional of layer thickness by gradient descent (Sections 4). Finally, numerical examples of model validation, measurement approximation and thickness reconstruction are given in Section (5). For the use of asymptotic models in inverse problems we may cite [8], [15] and [16] for various applications.

\section{Asymptotic approximation of axisymmetric eddy current model}

This section is devoted to a formal derivation of asymptotic models for eddy current problems with the presence of highly conducting thin deposits. The objective is to get the effective transmission conditions on the interface between the thin layer and the tube with which the variational asymptotic model has no longer the volume integral on the thin layer domain.

First let us briefly introduce the axisymmetric eddy current problem. For more details readers may refer to [10]. In the cylindrical coordinates, a vector field $\boldsymbol{a}$ can be decomposed into the meridian part $\boldsymbol{a}_{m}=a_{r} \boldsymbol{e}_{r}+a_{z} \boldsymbol{e}_{z}$ and the azimuthal part $\boldsymbol{a}_{\theta}=a_{\theta} \boldsymbol{e}_{\theta} . \quad \boldsymbol{a}$ is axisymmetric if $\partial_{\theta} \boldsymbol{a}$ vanishes. Under the assumption of axisymmetry and the low electric permittivity / low frequency regime $(\omega \epsilon \ll \sigma)$, the 3-D time-harmonic Maxwell's equations for the electric and magnetic fields $(\boldsymbol{E}, \boldsymbol{H})$

$$
\left\{\begin{aligned}
\operatorname{curl} \boldsymbol{H}+(\mathrm{i} \omega \epsilon-\sigma) \boldsymbol{E}=\boldsymbol{J} & \text { in } \mathbb{R}^{3}, \\
\operatorname{curl} \boldsymbol{E}-\mathrm{i} \omega \mu \boldsymbol{H}=0 & \text { in } \mathbb{R}^{3},
\end{aligned}\right.
$$

with a divergence-free axisymmetric applied source $\boldsymbol{J}(\operatorname{div} \boldsymbol{J}=0)$ can be reduced to a second order equation on a 2-D domain $\mathbb{R}_{+}^{2}:=\{(r, z): r \geq 0, z \in \mathbb{R}\}$ for the azimuthal part of the electric field $E_{\theta}$ that we denote in the sequel by $u=E_{\theta}$ :

$$
-\operatorname{div}\left(\frac{1}{\mu r} \nabla(r u)\right)-\mathrm{i} \omega \sigma u=\mathrm{i} \omega J_{\theta}=\mathrm{i} \omega J \quad \text { in } \mathbb{R}_{+}^{2},
$$

where $\nabla:=\left(\partial_{r}, \partial_{z}\right)^{t}$ and div $:=\nabla \cdot$ are gradient and divergence operators in 2-D Cartesian coordinates. Assume that $J \in L^{2}\left(\mathbb{R}_{+}^{2}\right)$ has compact support, and that $\mu$ and $\sigma$ are in $L^{\infty}\left(\mathbb{R}_{+}^{2}\right)$ such that $\mu \geq \mu_{v}>0$, $\sigma \geq 0$ and that $\mu=\mu_{v}, \sigma=0$ for $r \geq r_{0}$ sufficiently large. Then problem (11) with a decay condition at infinity $\left(u \rightarrow 0\right.$ as $\left.r^{2}+z^{2} \rightarrow 0\right)$ has a unique solution in $H\left(\mathbb{R}_{+}^{2}\right)$ where for any $\Omega \subset \mathbb{R}_{+}^{2}$ we denote

$$
H(\Omega):=\left\{v: r^{1 / 2}\left(1+r^{2}\right)^{-\lambda / 2} v \in L^{2}(\Omega), r^{-1 / 2} \nabla(r v) \in L^{2}(\Omega)\right\}
$$

with $\lambda$ any real $>1$ (see [10]). Let us indicate that if $\Omega$ is bounded in the $r$-direction then $H(\Omega)$ is equivalent to the following space for which we shall use the same notation

$$
H(\Omega):=\left\{v: r^{1 / 2} v \in L^{2}(\Omega), r^{-1 / 2} \nabla(r v) \in L^{2}(\Omega)\right\}
$$


Hence, the eddy current equation (1) writes in the variational form

$$
a(u, v):=\int_{\Omega}\left(\frac{1}{\mu r} \nabla(r u) \cdot \nabla(r \bar{v})-\mathrm{i} \omega \sigma r w \bar{v}\right) \mathrm{d} r \mathrm{~d} z=\int_{\Omega} \mathrm{i} \omega J \bar{v} r \mathrm{~d} r \mathrm{~d} z \quad \forall v \in H(\Omega) .
$$

For numerical reasons, the computational domain will be truncated in radial direction at $r=r_{*}$ with $r_{*}>0$ sufficiently large, and we set a Neumann boundary condition on $r=r_{*}$. The solution to the truncated problem should satisfy (1) on $\Omega=B_{r_{*}}:=\left\{(r, z) \in \mathbb{R}^{2}: 0 \leq r \leq r_{*}\right\}$. This is why we shall use in the sequel the generic notation for the variational space $H(\Omega)$ with $\Omega$ denoting $\mathbb{R}_{+}^{2}$ or $B_{r_{*}}$. We also recall that the problem on $\Omega=B_{r_{*}}$ can be equivalently truncated to a bounded domain $B_{r_{*}, z_{*}}=\left\{(r, z) \in \mathbb{R}^{2}: 0 \leq r \leq r_{*},|z|<z_{*}\right\}$ by introducing appropriate Dirichlet-to-Neumann operators on the boundaries $\Gamma_{ \pm}:=\left\{(r, z): 0 \leq r \leq r_{*}, z=\right.$ $\left.\pm z_{*}\right\}$. This would be convenient for accelerating numerical evaluation of the solution. The analysis and examples of domain truncation using Neumann and Dirichlet-to-Neumann boundary conditions can be found in [10. We remark that the derivation of the asymptotic models and the inversion methods in the sequel are independent of the domain truncation strategy. This is why we use the variational formulation (2) as our starting point and then we apply domain truncation in the numerical examples.

Finally let us specify the problem settings of eddy current testing for thin layer deposit. We consider a thin copper deposit covering axisymmetrically the shell side of the tube (see Figure 1 for a radial cut of the setting in the cylindrical coordinate system, the $z$-axis is the tube axis, the thin deposit shown in blue is exaggerated in thickness). The eddy current probe is composed of two coaxial coils, whose radial cuts are represented by two small rectangles in Figure 1 , that move in the $z$-direction during a scan.

On the domain of problem $\Omega$, we denote $\Omega_{ \pm}:=\left\{(r, z) \in \Omega: r \gtrless r_{t_{2}}\right\}$. The applied current $J$ is supported by the coils, therefore supp $J \subset \Omega_{-}$. The deposit layer is depicted by $\Omega_{c}^{\delta} \subset \Omega_{+}$with a variable thickness $f_{\delta}(z)$ (exaggerated in thickness, shown in blue). We denote by $u_{ \pm}^{\delta}$ the electric fields outside the deposit layer, with $u_{-}^{\delta}$ on $\Omega_{-}$and $u_{+}^{\delta}$ on $\Omega_{+} \backslash \Omega_{c}^{\delta}$, and by $u_{c}^{\delta}$ the in-layer electric field, i.e. on $\Omega_{c}^{\delta}$.

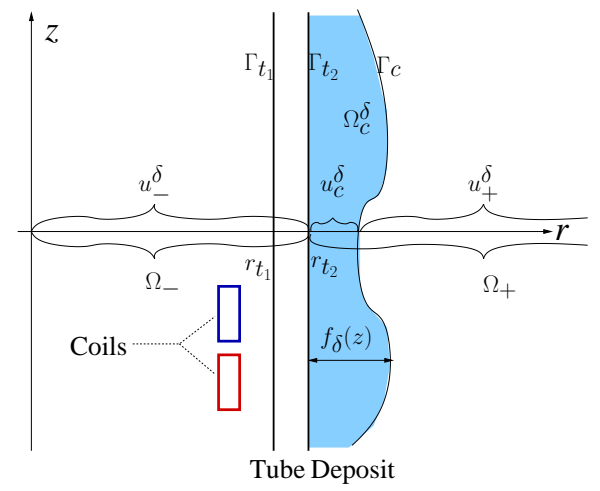

Figure 1: Representation of the eddy current testing of a thin layer deposit.

\subsection{Rescaled in-layer eddy current equation}

The first step is to rewrite the in-layer eddy current equation by rescaling the coordinate in the transverse direction and the conductivity with respect to the layer thickness. The analytical solution of this rescaled equation allows to get a relation between the boundary values of Dirichlet and Neumann type on the two longitudinal boundaries of the thin layer.

We assume that the thickness $f_{\delta}(z)$ writes $f_{\delta}(z)=\delta d(z)$, with $\delta>0$ a small parameter and $d(z)>0$ a dimensionless quantity. As we are concerned only with the $\mathcal{Z}_{1, n}(n=0,1)$ transmission conditions (see [9]), we rescale the deposit conductivity by $\sigma_{c}=\sigma_{1} / \delta$, where the rescaled conductivity $\sigma_{1}$ is considerably smaller than $\sigma_{c}$. We rescale also the coordinate in the transverse direction of the deposits by the change of variable $\rho=\left(r-r_{t_{2}}\right) / \delta$ with $\rho \in[0, d(z)]$, and we denote by $\tilde{u}(\rho, z):=u_{c}^{\delta}\left(r_{t_{2}}+\delta \rho, z\right)$ the rescaled in-layer solution. Let $k_{1}$ be such that $\left(k_{1}\right)^{2}=\mathrm{i} \omega \mu_{c} \sigma_{1}$. From (1) and the rescaling settings, we obtain

$$
\partial_{\rho}^{2} \tilde{u}=-\delta \mathcal{B}_{1}^{1} \tilde{u}-\delta^{2} \mathcal{B}_{1}^{2} \tilde{u}-\delta^{3} \mathcal{B}_{1}^{3} \tilde{u}-\delta^{4} \mathcal{B}_{1}^{4} \tilde{u}
$$


with the operators

$$
\begin{array}{rlrl}
\mathcal{B}_{1}^{1} & =\frac{2 \rho}{r_{t_{2}}} \partial_{\rho}^{2}+\frac{1}{r_{t_{2}}} \partial_{\rho}+k_{1}^{2}, & \mathcal{B}_{1}^{2} & =\frac{\rho^{2}}{r_{t_{2}}^{2}} \partial_{\rho}^{2}+\frac{\rho}{r_{t_{2}}^{2}} \partial_{\rho}-\frac{1}{r_{t_{2}}^{2}}+\partial_{z}^{2}+\frac{2 \rho}{r_{t_{2}}} k_{1}^{2}, \\
\mathcal{B}_{1}^{3} & =\frac{2 \rho}{r_{t_{2}}} \partial_{z}^{2}+\frac{\rho^{2}}{r_{t_{2}}^{2}} k_{1}^{2}, & \mathcal{B}_{1}^{4}=\frac{\rho^{2}}{r_{t_{2}}^{2}} \partial_{z}^{2} .
\end{array}
$$

\subsection{Transmission conditions between in-layer field and fields outside the layer}

The transmission conditions between the field inside the tube $u_{-}^{\delta}$ and the in-layer field $u_{c}^{\delta}$ are

$$
u_{-}^{\delta}=u_{c}^{\delta} \quad \text { and } \quad \mu_{t}^{-1} \partial_{r}\left(r u_{-}^{\delta}\right)=\mu_{c}^{-1} \partial_{r}\left(r u_{c}^{\delta}\right) \quad \text { on } \quad \Gamma_{t_{2}} .
$$

The transmission conditions between $u_{c}^{\delta}$ and the field outside the deposit layer $u_{+}^{\delta}$ write

$$
u_{+}^{\delta}=u_{c}^{\delta} \quad \text { and } \quad \mu_{v}^{-1} \partial_{n}\left(r u_{+}^{\delta}\right)=\mu_{c}^{-1} \partial_{n}\left(r u_{c}^{\delta}\right) \quad \text { on } \quad \Gamma_{c} .
$$

With the unit normal and tangential vectors on $\Gamma_{c}$ at the point $\left(r_{t_{2}}+\delta d(z), z\right)$

$$
\boldsymbol{n}=\frac{\left(1,-\delta d^{\prime}(z)\right)}{\sqrt{1+\left(\delta d^{\prime}(z)\right)^{2}}} \quad \text { and } \quad \boldsymbol{\tau}=\frac{\left(\delta d^{\prime}(z), 1\right)}{\sqrt{1+\left(\delta d^{\prime}(z)\right)^{2}}}
$$

we rewrite (5) using vector decomposition on these directions $(\boldsymbol{n}, \boldsymbol{\tau})$

$$
u_{c}^{\delta}=u_{+}^{\delta} \quad \text { and } \quad \partial_{r}\left(r u^{\delta}\right)=\frac{\mu_{c} / \mu_{v}+\left(\delta d^{\prime}\right)^{2}}{1+\left(\delta d^{\prime}\right)^{2}} \partial_{r}\left(r u_{+}^{\delta}\right)+\left(1-\mu_{c} / \mu_{v}\right) \frac{\delta d^{\prime}}{1+\left(\delta d^{\prime}\right)^{2}} \partial_{z}\left(r u_{+}^{\delta}\right) \quad \text { on } \Gamma_{c} .
$$

Together with the partial differential equation for the in-layer field (3), these transmission conditions on both sides of the thin layer as boundary conditions will serve to establish Cauchy problems whose solutions link the fields on both sides of the thin layer, that is a relationship between $u_{-}^{\delta}$ on $\Gamma_{t_{2}}$ and $u_{+}^{\delta}$ on $\Gamma_{c}$. To get the effective transmission conditions on $\Gamma_{t_{2}}$, we further apply a Taylor expansion of $u_{+}^{\delta}$.

\subsection{Extension of $u_{+}^{\delta}$ using Taylor's expansions}

To get effective transmission conditions linking up $u_{ \pm}^{\delta}$ on the interface $\Gamma_{t_{2}}$ between tube and thin deposit, we should extend the field $u_{+}^{\delta}$, originally defined only on $\Omega_{+} \backslash \Omega_{c}$, to the whole domain $\Omega_{+}$. A natural approach would be to assume that the extended field, still denoted by $u_{+}^{\delta}$, satisfies the same eddy current equation (11) as $u_{+}^{\delta}$ on $\Omega_{+} \backslash \Omega_{c}$, i.e. with coefficients of the vacuum $\mu=\mu_{v}, \sigma=\sigma_{v}=0$. We remark that the right hand side of equation (1) vanishes in $\Omega_{+}$since $J$ has support only in $\Omega_{-}$. Using the variable substitution $\nu=r-r_{t_{2}}$, one rewrites (1) in $\Omega_{+}$as

$$
\sum_{j=0}^{4} \nu^{j} \mathcal{A}_{j}\left(\nu \partial_{\nu}, \partial_{z}\right) u_{+}^{\delta}=0
$$

where

$$
\begin{aligned}
& \mathcal{A}_{0}\left(\nu \partial_{\nu}, \partial_{z}\right)=\left(\nu \partial_{\nu}\right)^{2}-\nu \partial_{\nu}, \quad \mathcal{A}_{1}\left(\nu \partial_{\nu}, \partial_{z}\right)=\frac{2}{r_{t_{2}}}\left(\nu \partial_{\nu}\right)^{2}-\frac{1}{r_{t_{2}}} \nu \partial_{\nu}, \\
& \mathcal{A}_{2}\left(\nu \partial_{\nu}, \partial_{z}\right)=\frac{1}{r_{t_{2}}^{2}}\left(\nu \partial_{\nu}\right)^{2}-\frac{1}{r_{t_{2}}^{2}}+\partial_{z}^{2}, \quad \mathcal{A}_{3}\left(\nu \partial_{\nu}, \partial_{z}\right)=\frac{2}{r_{t_{2}}} \partial_{z}^{2}, \quad \quad \mathcal{A}_{4}\left(\nu \partial_{\nu}, \partial_{z}\right)=\frac{1}{r_{t_{2}}^{2}} \partial_{z}^{2} .
\end{aligned}
$$

The asymptotic expansion of $u_{+}^{\delta}$ with respect to $\delta$ is in the form $u_{+}^{\delta}(r, z)=\sum_{n=0}^{\infty} \delta^{n} u_{n}^{+}(r, z)$. Obviously each term $u_{n}^{+}(r, z)$ verifies the same equation (7). With Taylor series expansion, one has

$$
u_{n}^{+}\left(r_{t_{2}}+\nu, z\right)=\sum_{k=0}^{\infty} \nu^{k} u_{n, k}^{+}(z) \quad \text { where } \quad u_{n, k}^{+}(z)=\frac{1}{k !}\left(\partial_{\nu}^{k} u_{n}^{+}\right)\left(r_{t_{2}}, z\right) .
$$


Since $\nu \partial_{\nu}\left(\nu^{k} u_{n, k}^{+}(z)\right)=k\left(\nu^{k} u_{n, k}^{+}(z)\right)$, we can indeed write $\mathcal{A}_{i}\left(\nu \partial_{\nu}, \partial_{z}\right)$ as $\mathcal{A}_{i}\left(k, \partial_{z}\right)$ while it is applied to $\left(\nu^{k} u_{n, k}^{+}(z)\right)$. Thus, from (7) one has $\sum_{j=0}^{4} \sum_{k=0}^{\infty} \mathcal{A}_{j}\left(k, \partial_{z}\right)\left(\nu^{k+j} u_{n, k}^{+}\right)=0$. The equality at order $\mathcal{O}\left(\nu^{k}\right)$ gives

$$
\mathcal{A}_{0}\left(k, \partial_{z}\right) u_{n, k}^{+}=-\sum_{j=1}^{4} \mathcal{A}_{j}\left(k-j, \partial_{z}\right) u_{n, k-j}^{+},
$$

with $u_{n,-1}^{+}=u_{n,-2}^{+}=u_{n,-3}^{+}=u_{n,-4}^{+}=0$. Now we consider $\mathcal{A}_{0}\left(k, \partial_{z}\right)=k^{2}-k$. For $k \geq 2, \mathcal{A}_{0}\left(k, \partial_{z}\right) \neq 0$, thus invertible with its inverse $\mathcal{A}_{0}^{-1}\left(k, \partial_{z}\right)=\frac{1}{k^{2}-k}$. So we have

$$
u_{n, k}^{+}=-\mathcal{A}_{0}^{-1}\left(k, \partial_{z}\right)\left(\sum_{j=1}^{4} \mathcal{A}_{j}\left(k-j, \partial_{z}\right) u_{n, k-j}^{+}\right), \quad k \geq 2 .
$$

Now we inductively define two families of operators $\left\{\mathcal{S}_{k}^{0}\left(\partial_{z}\right), \mathcal{S}_{k}^{1}\left(\partial_{z}\right)\right\}$ :

$$
k \geq 2\left\{\begin{array}{l}
\mathcal{S}_{0}^{0}:=\mathrm{Id}, \quad \mathcal{S}_{0}^{1}:=0, \quad \mathcal{S}_{1}^{0}:=0, \quad \mathcal{S}_{1}^{1}:=\mathrm{Id}, \\
\mathcal{S}_{k}^{0}:=-\mathcal{A}_{0}^{-1}\left(k, \partial_{z}\right)\left(\sum_{j=1}^{4} \mathcal{A}_{j}\left(k-j, \partial_{z}\right) \mathcal{S}_{k-j}^{0}\left(\partial_{z}\right)\right), \\
\mathcal{S}_{k}^{1}:=-\mathcal{A}_{0}^{-1}\left(k, \partial_{z}\right)\left(\sum_{j=1}^{4} \mathcal{A}_{j}\left(k-j, \partial_{z}\right) \mathcal{S}_{k-j}^{1}\left(\partial_{z}\right)\right) .
\end{array}\right.
$$

From the induction (8) one gets $u_{n, k}^{+}(z)=\mathcal{S}_{k}^{0}\left(\partial_{z}\right) u_{n}^{+}\left(r_{t_{2}}, z\right)+\mathcal{S}_{k}^{1}\left(\partial_{z}\right) \partial_{r} u_{n}^{+}\left(r_{t_{2}}, z\right)$. Therefore we have the following expansion

$$
\left\{\begin{aligned}
u_{n}^{+}\left(r_{t_{2}}+\nu, z\right) & =\sum_{k=0}^{\infty} \nu^{k}\left(\mathcal{S}_{k}^{0}\left(\partial_{z}\right) u_{n}^{+}+\mathcal{S}_{k}^{1}\left(\partial_{z}\right) \partial_{r} u_{n}^{+}\right)\left(r_{t_{2}}, z\right) \\
\partial_{r} u_{n}^{+}\left(r_{t_{2}}+\nu, z\right) & =\sum_{k=0}^{\infty} \nu^{k}(k+1)\left(\mathcal{S}_{k+1}^{0}\left(\partial_{z}\right) u_{n}^{+}+\mathcal{S}_{k+1}^{1}\left(\partial_{z}\right) \partial_{r} u_{n}^{+}\right)\left(r_{t_{2}}, z\right) .
\end{aligned}\right.
$$

We also define the operators $\widetilde{\mathcal{S}}_{k}^{0}:=\mathcal{S}_{k}^{0}-\frac{1}{r_{t_{2}}} \mathcal{S}_{k}^{1}$ and $\widetilde{\mathcal{S}}_{k}^{1}:=\frac{1}{r_{t_{2}}} \mathcal{S}_{k}^{1}$. Then the above Taylor expansions write

$$
\left\{\begin{aligned}
u_{n}^{+}\left(r_{t_{2}}+\nu, z\right) & =\sum_{k=0}^{\infty} \nu^{k}\left(\widetilde{\mathcal{S}}_{k}^{0}\left(\partial_{z}\right) u_{n}^{+}+\widetilde{\mathcal{S}}_{k}^{1} \partial_{r}\left(r u_{n}^{+}\right)\right)\left(r_{t_{2}}, z\right) \\
\partial_{r}\left(r u_{n}^{+}\right)\left(r_{t_{2}}+\nu, z\right) & =\sum_{k=0}^{\infty} \nu^{k}(k+1)\left(\left(r_{t_{2}} \widetilde{\mathcal{S}}_{k+1}^{0}+\widetilde{\mathcal{S}}_{k}^{0}\right) u_{n}^{+}+\left(r_{t_{2}} \widetilde{\mathcal{S}}_{k+1}^{1}+\widetilde{\mathcal{S}}_{k}^{1}\right) \partial_{r}\left(r u_{n}^{+}\right)\right)\left(r_{t_{2}}, z\right) .
\end{aligned}\right.
$$

Although only asymptotic problems of order 0 and 1 are discussed in the sequel, the above expressions for any orders could facilitate further exploitations beyond the scope of the current work.

\section{Asymptotic models for deposits with variable layer thickness}

In this section, we build the asymptotic models of order 0 and 1 for thin deposits of variable thickness, that is to establish the transmission conditions $\mathcal{Z}_{1, n}$ for $n=0,1$ based on the material of the previous section. The general procedure consists in solving analytically a Cauchy problem of the rescaled in-layer eddy current equation in its asymptotic expansion form (3) with boundary conditions on $\Gamma_{t_{2}}$ (4). The resulting solution yields the Dirichlet and Neumann boundary values on $\Gamma_{c}$ which should match with transmission conditions (6). Finally with the Taylor expansions of $u_{+}^{\delta}$ (10), one gets the effective transmission conditions on $\Gamma_{t_{2}}$.

To simplify the presentation, especially the complexities introduced by the transmission conditions (6) on the curved boundary $\Gamma_{c}$, we assume that the magnetic permeability of the deposits equals to that of vacuum $\mu_{c}=\mu_{v}$. This assumption matches the practical application we have in mind since the relative permeability of copper is 1 . 
We introduce the notation for jump and mean values on $\Gamma_{t_{2}}$

$$
\begin{array}{rlrl}
{[v]:=v_{+}\left|r_{t_{2}}-v_{-}\right|_{r_{2}},} & {\left[\mu^{-1} \partial_{r}(r v)\right]} & =\left.\mu_{v}^{-1} \partial_{r}\left(r v_{+}\right)\right|_{r_{t_{2}}}-\left.\mu_{t}^{-1} \partial_{r}\left(r v_{-}\right)\right|_{r_{t_{2}}}, \\
\langle v\rangle:=\frac{1}{2}\left(v_{+}\left|r_{t_{2}}+v_{-}\right| r_{t_{2}}\right) & \left\langle\mu^{-1} \partial_{r}(r v)\right\rangle=\frac{1}{2}\left(\left.\mu_{v}^{-1} \partial_{r}\left(r v_{+}\right)\right|_{r_{t_{2}}}+\left.\mu_{t}^{-1} \partial_{r}\left(r v_{-}\right)\right|_{r_{t_{2}}}\right) .
\end{array}
$$

\subsection{Formal derivation of $\mathcal{Z}_{1,0}$ transmission conditions}

We recall the asymptotic expansion $u_{+}^{\delta}=\sum_{n=0}^{\infty} \delta^{n} u_{n}^{+}$and expand $\tilde{u}(\rho, z)=\sum_{n=0}^{\infty} \delta^{n} u_{n}(\rho, z)$ and $u_{-}^{\delta}=$ $\sum_{n=0}^{\infty} \delta^{n} u_{n}^{-}$. The eddy current equation (3) and the transmission conditions (4) on $\Gamma_{t_{2}}$ for the first term $u_{0}$ yield the Cauchy problem

$$
\left\{\begin{array}{l}
\partial_{\rho}^{2} u_{0}=0 \quad \rho \in[0, d(z)] \\
\left.u_{0}\right|_{\rho=0}=\left.u_{0}^{-}\right|_{r_{t_{2}}},\left.\quad \partial_{\rho} u_{0}\right|_{\rho=0}=0
\end{array}\right.
$$

This problem has a constant solution $u_{0}(\rho, z)=\left.u_{0}^{-}\right|_{r_{t_{2}}}$ for $\rho \in[0, d(z)]$. Considering its value at $\rho=d(z)$ and the first transmission condition of (6) for $u_{0}$ on $\Gamma_{c}$, we get

$$
\left.u_{0}^{-}\right|_{r_{t_{2}}}=\left.u_{0}^{+}\right|_{r_{t_{2}}}
$$

The Cauchy problem for $u_{1}$ with initial values given by (4) is

$$
\left\{\begin{array}{l}
\partial_{\rho}^{2} u_{1}=-\mathcal{B}_{1}^{1} u_{0}=-\left.k_{1}^{2} u_{0}\right|_{r_{t_{2}}} \quad \rho \in[0, d(z)], \\
\left.u_{1}\right|_{\rho=0}=\left.u_{1}^{-}\right|_{r_{t_{2}}}, \\
\left.\partial_{\rho} u_{1}\right|_{\rho=0}=-\left.\frac{1}{r_{t_{2}}} u_{0}^{-}\right|_{r_{t_{2}}}+\left.\frac{1}{r_{t_{2}}} \frac{\mu_{c}}{\mu_{t}} \partial_{r}\left(r u_{0}^{-}\right)\right|_{r_{t_{2}}} .
\end{array}\right.
$$

It follows that

$$
\begin{aligned}
& \partial_{\rho} u_{1}=-\frac{1}{r_{t_{2}}}\left(\left.u_{0}^{-}\right|_{r_{t_{2}}}-\left.\frac{\mu_{c}}{\mu_{t}} \partial_{r}\left(r u_{0}^{-}\right)\right|_{r_{t_{2}}}\right)-\left.\rho k_{1}^{2} u_{0}^{-}\right|_{r_{t_{2}}}, \\
& u_{1}=\left.u_{1}^{-}\right|_{r_{t_{2}}}-\frac{\rho}{r_{t_{2}}}\left(\left.u_{0}^{-}\right|_{r_{t_{2}}}-\left.\frac{\mu_{c}}{\mu_{t}} \partial_{r}\left(r u_{0}^{-}\right)\right|_{r_{t_{2}}}\right)-\left.\frac{\rho^{2}}{2} k_{1}^{2} u_{0}^{-}\right|_{r_{t_{2}}}
\end{aligned}
$$

The second transmission condition of (6) for $u_{1}$ on $\Gamma_{c}$ implies

$$
\left.\partial_{\rho} u_{1}\right|_{\rho=d(z)}=-\frac{1}{r_{t_{2}}}\left(\left.u_{0}^{+}\right|_{r_{t_{2}}}-\left.\frac{\mu_{c}}{\mu_{v}} \partial_{r}\left(r u_{0}^{+}\right)\right|_{r_{t_{2}}}\right) .
$$

Matching (14) and (12) at $\rho=d(z)$ gives

$$
\left.\frac{1}{r_{t_{2}}} \frac{\mu_{c}}{\mu_{t}} \partial_{r}\left(r u_{0}^{-}\right)\right|_{r_{t_{2}}}-\left.k_{1}^{2} d(z) u_{0}^{-}\right|_{r_{t_{2}}}=\left.\frac{1}{r_{t_{2}}} \frac{\mu_{c}}{\mu_{v}} \partial_{r}\left(r u_{0}^{+}\right)\right|_{r_{t_{2}}} .
$$

Equalities (11), (15) and the fact that $k_{1}^{2}=\mathrm{i} \omega \sigma_{1} \mu_{c}$ imply that

$$
\begin{aligned}
& {\left[u_{0}\right]=0 \quad \text { and } \quad\left[\mu^{-1} \partial_{r}\left(r u_{0}\right)\right]=-\mathrm{i} \gamma_{1}\left\langle u_{0}\right\rangle} \\
& \text { where } \quad \gamma_{1}=\omega \sigma_{1} d(z) r_{t_{2}}=\omega \sigma_{c} f_{\delta}(z) r_{t_{2}} .
\end{aligned}
$$

\subsection{Asymptotic model of order 0}

If $u^{\delta}:=u_{ \pm}^{\delta}$ in $\Omega_{ \pm}$denote an approximation of the exact solution up to $O(\delta)$ error, then from (16), possible transmission conditions can set as

$$
\left[u^{\delta}\right]=0 \quad \text { and } \quad\left[\mu^{-1} \partial_{r}\left(r u^{\delta}\right)\right]=-\mathrm{i} \gamma_{1}\left\langle u^{\delta}\right\rangle
$$


The first condition of (17) implies the continuity of $u^{\delta}$ through $\Gamma_{t_{2}}$. Moreover, $u^{\delta}$ satisfies

$$
-\operatorname{div}\left(\frac{1}{\mu r} \nabla\left(r u^{\delta}\right)\right)-\mathrm{i} \omega \sigma u^{\delta}=\mathrm{i} \omega J \quad \text { in } \Omega_{ \pm},
$$

We shall prove that the above equations define a well posed problem. The variational formulation associated with (18) and (17) is

$$
\begin{aligned}
a_{1,0}\left(u^{\delta}, v\right) & =\int_{\Omega} \mathrm{i} \omega J \bar{v} r \mathrm{~d} r \mathrm{~d} z \quad \forall v \in H(\Omega), \\
\text { with } \quad a_{1,0}\left(u^{\delta}, v\right) & :=\int_{\Omega_{+} \cup \Omega_{-}}\left(\frac{1}{\mu r} \nabla\left(r u^{\delta}\right) \cdot \nabla(r \bar{v})-\mathrm{i} \omega \sigma u^{\delta} \bar{v} r\right) \mathrm{d} r \mathrm{~d} z-\int_{\Gamma_{t_{2}}} \mathrm{i} \gamma_{1} u^{\delta} \bar{v} \mathrm{~d} s .
\end{aligned}
$$

Proposition 3.1. Assume that the source $J \in L_{1 / 2}^{2}(\Omega)$ has compact support, that the permeability $\mu>0$ and the conductivity $\sigma \geq 0$ are piecewise constant and bounded in $\Omega$. Assume in addition that there exist $0<\mu_{\mathrm{inf}}<\mu_{\mathrm{sup}}<+\infty$ such that $\mu$ satisfies $\mu_{\mathrm{inf}}<\mu<\mu_{\mathrm{sup}}$. Then the variational asymptotic problem (19) has a unique solution $u^{\delta}$ in $H(\Omega)$.

Proof. One verifies that $a_{1,0}$ is a continuous sesquilinear form. It is sufficient to show that $a_{1,0}$ is coercive

$$
\Re\left(a_{1,0}\left(u^{\delta}, u^{\delta}\right)\right) \geq \int_{\Omega_{+} \cup \Omega_{-}} \frac{1}{\mu r}\left|\nabla\left(r u^{\delta}\right)\right|^{2} \mathrm{~d} r \mathrm{~d} z \geq \frac{1}{\mu_{\text {sup }}}\left\|r^{-1 / 2} \nabla\left(r u^{\delta}\right)\right\|_{L^{2}(\Omega)}^{2} \geq \frac{C}{\mu_{\text {sup }}}\left\|u^{\delta}\right\|_{H(\Omega)}^{2} .
$$

for some postive constant $C$, where the last inequality is due to a Poincaré-type inequality (see [10, Lemma 2.1] for $\Omega=\mathbb{R}_{+}^{2}$ or [10, inequality (17)] for $\Omega=B_{r_{*}}$ ). Therefore, we conclude from the Lax-Milgram Theorem the existence and uniqueness of $u^{\delta} \in H(\Omega)$ to problem (19).

\subsection{Formal derivation of $\mathcal{Z}_{1,1}$ transmission conditions}

We pursue the formal calculations of Section 3.1 till the following order. With Taylor expansions (10), the first transmission condition of (6) for $u_{1}$ writes explicitly on $\Gamma_{t_{2}}$

$$
\left.u_{1}\right|_{\rho=d(z)}=\left.u_{1}^{+}\right|_{r_{t_{2}}}+\left.\frac{d(z)}{r_{t_{2}}}\left(-u_{0}^{+}+\partial_{r}\left(r u_{0}^{+}\right)\right)\right|_{r_{t_{2}}} .
$$

The above equality and (13) with $\rho=d(z)$ yield

$$
\left.u_{1}^{+}\right|_{r_{t_{2}}}=\left.u_{1}^{-}\right|_{r_{t_{2}}}+\left.\frac{\mathrm{i} \omega \sigma_{1} \mu_{c} d^{2}}{2} u_{0}^{-}\right|_{r_{t_{2}}} .
$$

Then we consider the Cauchy problem for $u_{2}$ derived from the asymptotic development (3) and the transmission conditions (4) on $\Gamma_{t_{2}}$ for $u_{2}$

$$
\left\{\begin{aligned}
& \partial_{\rho}^{2} u_{2}=-\mathcal{B}_{1}^{1} u_{1}-\mathcal{B}_{1}^{2} u_{0} \\
&=-\left.k_{1}^{2} u_{1}\right|_{r_{t_{2}}}+\left.\left(\frac{2}{r_{t_{2}}^{2}}-\partial_{z}^{2}\right) u_{0}^{-}\right|_{r_{t_{2}}}-\left.\frac{1}{r_{t_{2}}^{2}} \frac{\mu_{c}}{\mu_{t}} \partial_{r}\left(r u_{0}^{-}\right)\right|_{r_{t_{2}}} \\
&+\rho k_{1}^{2}\left(\left.\frac{2}{r_{t_{2}}} u_{0}^{-}\right|_{r_{t_{2}}}-\left.\frac{1}{r_{t_{2}}} \frac{\mu_{c}}{\mu_{t}} \partial_{r}\left(r u_{0}^{-}\right)\right|_{r_{t_{2}}}\right)+\left.\rho^{2} \frac{k_{1}^{4}}{2} u_{0}^{-}\right|_{r_{t_{2}}} \quad \rho \in[0, d(z)] \\
&\left.u_{2}\right|_{\rho=0}=\left.u_{2}^{-}\right|_{r_{t_{2}}}, \\
&\left.\partial_{\rho} u_{2}\right|_{\rho=0}=-\left.\frac{1}{r_{t_{2}}} u_{1}^{-}\right|_{r_{t_{2}}}+\left.\frac{1}{r_{t_{2}}} \frac{\mu_{c}}{\mu_{t}} \partial_{r}\left(r u_{1}^{-}\right)\right|_{r_{t_{2}}} .
\end{aligned}\right.
$$

We get

$$
\begin{aligned}
\partial_{\rho} u_{2} & =-\frac{1}{r_{t_{2}}}\left(\left.u_{1}^{-}\right|_{r_{t_{2}}}-\left.\frac{\mu_{c}}{\mu_{t}} \partial_{r}\left(r u_{1}^{-}\right)\right|_{r_{t_{2}}}\right)+\rho\left(-\left.k_{1}^{2} u_{1}^{-}\right|_{t_{t_{2}}}+\left.\left(\frac{2}{r_{t_{2}}^{2}}-\partial_{z}^{2}\right) u_{0}^{-}\right|_{r_{t_{2}}}-\left.\frac{1}{r_{t_{2}}^{2}} \frac{\mu_{c}}{\mu_{t}} \partial_{r}\left(r u_{0}^{-}\right)\right|_{r_{t_{2}}}\right) \\
& +\rho^{2} \frac{k_{1}^{2}}{2}\left(\left.\frac{2}{r_{t_{2}}} u_{0}^{-}\right|_{r_{t_{2}}}-\left.\frac{1}{r_{t_{2}}} \frac{\mu_{c}}{\mu_{t}} \partial_{r}\left(r u_{0}^{-}\right)\right|_{r_{t_{2}}}\right)+\left.\rho^{3} \frac{k_{1}^{4}}{6} u_{0}^{-}\right|_{r_{t_{2}}} .
\end{aligned}
$$


Since $\mu_{c}=\mu_{v}$, the second transmission condition of (자) for $u_{2}$ on $\Gamma_{c}$ writes

$$
\left.\partial_{\rho} u_{2}\right|_{\rho=d(z)}=-\left.\frac{1}{r_{t_{2}}}\left(u_{1}^{+}-\partial_{r}\left(r u_{1}^{+}\right)\right)\right|_{r_{t_{2}}}+\left.d(z)\left(\left(\frac{2}{r_{t_{2}}^{2}}-\partial_{z}^{2}\right) u_{0}^{+}-\frac{1}{r_{t_{2}}^{2}} \partial_{r}\left(r u_{0}^{+}\right)\right)\right|_{r_{t_{2}}}
$$

The equality (23) with $\rho=d(z)$ and (24) yield

$$
\begin{aligned}
& \left.\frac{1}{\mu_{v}} \partial_{r}\left(r u_{1}^{+}\right)\right|_{r_{t_{2}}}=\left.\frac{1}{\mu_{t}} \partial_{r}\left(r u_{1}^{-}\right)\right|_{r_{t_{2}}}-\left.\mathrm{i} \omega \sigma_{1} r_{t_{2}} d(z) u_{1}^{-}\right|_{r_{t_{2}}} \\
& \quad+\left.\left(\frac{1}{2} \mathrm{i} \omega \sigma_{1} d(z)^{2}-\frac{1}{6} \omega^{2} \sigma_{1}^{2} \mu_{c} r_{t_{2}} d(z)^{3}\right) u_{0}^{-}\right|_{r_{t_{2}}}-\left.\frac{1}{2} \mathrm{i} \omega \sigma_{1} \mu_{c} d(z)^{2} \frac{1}{\mu_{t}} \partial_{r}\left(r u_{0}^{-}\right)\right|_{r_{t_{2}}} .
\end{aligned}
$$

From (11), (15), (22) and (25), one gets

$$
\begin{array}{ll} 
& {\left[u_{1}\right]=\mathrm{i} \gamma_{4}\left\langle u_{0}\right\rangle \text { and }\left[\mu^{-1} \partial_{r}\left(r u_{1}\right)\right]=-\mathrm{i} \gamma_{1}\left\langle u_{1}\right\rangle+\left(-\gamma_{2}+\mathrm{i} \gamma_{3}\right)\left\langle u_{0}\right\rangle-\mathrm{i} \gamma_{4}\left\langle\mu^{-1} \partial_{r}\left(r u_{0}\right)\right\rangle,} \\
\text { where } \quad \gamma_{2}=\frac{\omega^{2} \sigma_{1}^{2} \mu_{c} r_{t_{2}} d(z)^{3}}{6}=\frac{\omega^{2} \sigma_{c}^{2} \mu_{c} r_{t_{2}} f_{\delta}(z)^{3}}{6 \delta}, \quad \gamma_{3}=\frac{\omega \sigma_{1} d(z)^{2}}{2}=\frac{\omega \sigma_{c} f_{\delta}(z)^{2}}{2 \delta}, \\
\gamma_{4}=\frac{\omega \sigma_{1} \mu_{c} d(z)^{2}}{2}=\frac{\omega \sigma_{c} \mu_{c} f_{\delta}(z)^{2}}{2 \delta} .
\end{array}
$$

\subsection{Asymptotic model of order 1}

Let us still denote the solution of the asymptotic problem by $u^{\delta}=u_{ \pm}^{\delta}$ in $\Omega_{ \pm}$but assume that now it forms an approximation of the exact one up to $O\left(\delta^{2}\right)$ error. From (26), possible (natural) transmission conditions on $r=r_{t_{2}}$ can be written as

$$
\left[u^{\delta}\right]=\mathrm{i} \gamma_{4} \delta\left\langle u^{\delta}\right\rangle \quad \text { and } \quad\left[\mu^{-1} \partial_{r}\left(r u^{\delta}\right)\right]=\left(-\mathrm{i} \gamma_{1}-\gamma_{2} \delta+\mathrm{i} \gamma_{3} \delta\right)\left\langle u^{\delta}\right\rangle-\mathrm{i} \gamma_{4} \delta\left\langle\mu^{-1} \partial_{r}\left(r u^{\delta}\right)\right\rangle .
$$

However, in order to ensure the well-posedness of the asymptotic problem via variational approach, we are led to perturb these conditions with additional term of order $\mathcal{O}\left(\delta^{2}\right)$ in the first condition of (27) which is compatible with the desired order of approximation of the asymptotic model. More specifically we replace (27) with the following transmission conditions

$$
\begin{aligned}
& {\left[u^{\delta}\right]=\mathrm{i} \gamma_{4} \delta\left\langle u^{\delta}\right\rangle+\mathrm{i} \alpha \gamma_{5} \delta^{2}\left\langle\mu^{-1} \partial_{r}\left(r u^{\delta}\right)\right\rangle,} \\
& {\left[\mu^{-1} \partial_{r}\left(r u^{\delta}\right)\right]=\left(-\mathrm{i} \gamma_{1}-\gamma_{2} \delta+\mathrm{i} \gamma_{3} \delta\right)\left\langle u^{\delta}\right\rangle-\mathrm{i} \gamma_{4} \delta\left\langle\mu^{-1} \partial_{r}\left(r u^{\delta}\right)\right\rangle}
\end{aligned}
$$

where

$$
\gamma_{5}:=\frac{\omega \sigma_{c} \mu_{c}^{2}\left(f_{\delta}(z)+h(z)^{2}\right)^{3}}{r_{t_{2}} \delta^{2}},
$$

and $\alpha$ is a constant independent of $\delta$ to be fixed later. We remark that the first condition in (28) implies that $u^{\delta}$ is no longer continuous through $\Gamma_{t_{2}}$. Therefore, we consider the variational space

$$
H\left(\Omega_{-} \cup \Omega_{+}\right):=\left\{v:\left.v\right|_{\Omega_{ \pm}}=v_{ \pm} \in H\left(\Omega_{ \pm}\right)\right\} .
$$

The variational problem associated to (18) and (28) writes: for any $v \in H\left(\Omega_{-} \cup \Omega_{+}\right)$,

$$
a_{1,1}\left(u^{\delta}, v\right):=\int_{\Omega_{+} \cup \Omega_{-}}\left(\frac{1}{\mu r} \nabla\left(r u^{\delta}\right) \cdot \nabla(r \bar{v})-\mathrm{i} \omega \sigma u^{\delta} \bar{v} r\right) \mathrm{d} r \mathrm{~d} z+c_{1,1}\left(u^{\delta}, v\right)=\int_{\Omega} \mathrm{i} \omega J \bar{v} r \mathrm{~d} r \mathrm{~d} z
$$

where the term $c_{1,1}\left(u^{\delta}, v\right)$ should satisfy the following equation for regular $u_{ \pm}^{\delta}$

$$
c_{1,1}\left(u^{\delta}, v\right)=\int_{\Gamma_{t_{2}}}\left(\mu_{v}^{-1} \partial_{r}\left(r u_{+}^{\delta}\right) \bar{v}_{+}-\mu_{t}^{-1} \partial_{r}\left(r u_{-}^{\delta}\right) \bar{v}_{-}\right) \mathrm{d} s=\int_{\Gamma_{t_{2}}}\left(\left[\mu^{-1} \partial_{r}\left(r u^{\delta}\right)\right]\langle\bar{v}\rangle+\left\langle\mu^{-1} \partial_{r}\left(r u^{\delta}\right)\right\rangle[\bar{v}]\right) \mathrm{d} s .
$$

In fact, this is just the boundary term coming from integration by parts of the eddy current equation (11) in $\Omega_{ \pm}$multiplied by test functions $\bar{v}$. Hence, using the transmission conditions (28) we define $c_{1,1}(\cdot, \cdot)$ as

$$
c_{1,1}\left(u^{\delta}, v\right):=\int_{\Gamma_{t_{2}}}\left(\left(-\mathrm{i} \gamma_{1}+\frac{\mathrm{i} \gamma_{4}^{2}}{\alpha \gamma_{5}}-\gamma_{2} \delta+\mathrm{i} \gamma_{3} \delta\right)\left\langle u^{\delta}\right\rangle\langle\bar{v}\rangle-\frac{\gamma_{4}}{\alpha \gamma_{5} \delta}\left(\left\langle u^{\delta}\right\rangle[\bar{v}]+\left[u^{\delta}\right]\langle\bar{v}\rangle\right)-\frac{\mathrm{i}}{\alpha \gamma_{5} \delta^{2}}\left[u^{\delta}\right][\bar{v}]\right) \mathrm{d} s .
$$


Proposition 3.2. Let the layer thickness $f_{\delta}>0$. Under the same assumptions for $J, \mu$ and $\sigma$ as in Proposition [3.1, the variational problem (29) has a unique solution $u^{\delta} \in H\left(\Omega_{-} \cup \Omega_{+}\right)$if the coefficient $\alpha$ satisfies uniformly

$$
\alpha \geq \frac{2 \gamma_{4}^{2}}{\gamma_{5}\left(\gamma_{1}-\left(\gamma_{2}+\gamma_{3}\right) \delta\right)} \quad \forall z \in \mathbb{R},
$$

Remark 3.3. For the settings of physical parameters $\left(\omega, \sigma_{c}, \mu_{c}, r_{t_{2}}\right.$ and the interested range of the layer thickness $\left.0<f_{\delta}<0.1 \mathrm{~mm}\right)$, the coefficients satisfy $\gamma_{1}-\left(\gamma_{2}+\gamma_{3}\right) \delta>0$. The dimensionless positive quantity

$$
\frac{2 \gamma_{4}^{2}}{\gamma_{5}\left(\gamma_{1}-\left(\gamma_{2}+\gamma_{3}\right) \delta\right)}=\frac{1}{2-\left(\frac{\omega \sigma_{c} \mu_{c} f_{\delta}^{2}}{3}+\frac{f_{\delta}}{r_{t_{2}}}\right)}
$$

is monotonously increasing on $f_{\delta}$. It takes the maximum value $(\sim 0.54)$ at $f_{\delta}=0.1 \mathrm{~mm}$. Thus we could choose for example $\alpha=2 / 3$ in the following.

Proof. Obviously the sesquilinear form $a_{1,1}(\cdot, \cdot)$ is continuous on $H\left(\Omega_{-} \cup \Omega_{+}\right)^{2}$. It is sufficient to show that it is coercive on $H\left(\Omega_{-} \cup \Omega_{+}\right)$. For any $v \in H\left(\Omega_{-} \cup \Omega_{+}\right)$one has

$$
\begin{aligned}
a_{1,1}(v, v) & =\int_{\Omega_{+} \cup \Omega_{-}}\left(\frac{1}{\mu r}|\nabla(r v)|^{2}-\mathrm{i} \omega \sigma|v|^{2} r\right) \mathrm{d} r \mathrm{~d} z+c_{1,1}(v, v) \\
\text { with } \quad c_{1,1}(v, v) & =\int_{\Gamma_{t_{2}}}\left(\left(-\mathrm{i} \gamma_{1}+\frac{\mathrm{i} \gamma_{4}^{2}}{\alpha \gamma_{5}}-\gamma_{2} \delta+\mathrm{i} \gamma_{3} \delta\right)|\langle v\rangle|^{2}-\frac{2 \gamma_{4}}{\alpha \gamma_{5} \delta} \Re(\langle v\rangle[\bar{v}])-\frac{\mathrm{i}}{\alpha \gamma_{5} \delta^{2}}|[v]|^{2}\right) \mathrm{d} s .
\end{aligned}
$$

The real and imaginary parts of $c_{1,1}(v, v)$ are respectively

$$
\begin{aligned}
& \Re\left(c_{1,1}(v, v)\right)=\int_{\Gamma_{t_{2}}}\left(-\gamma_{2} \delta|\langle v\rangle|^{2}-\frac{2 \gamma_{4}}{\alpha \gamma_{5} \delta} \Re(\langle v\rangle[\bar{v}])\right) \mathrm{d} s, \\
& \Im\left(c_{1,1}(v, v)\right)=\int_{\Gamma_{t_{2}}}\left(\left(-\gamma_{1}+\frac{\gamma_{4}^{2}}{\alpha \gamma_{5}}+\gamma_{3} \delta\right)|\langle v\rangle|^{2}-\frac{1}{\alpha \gamma_{5} \delta^{2}}|[v]|^{2}\right) \mathrm{d} s .
\end{aligned}
$$

One has

$$
\begin{aligned}
\Re\left(c_{1,1}(v, v)\right)-\Im\left(c_{1,1}(v, v)\right) & =\int_{\Gamma_{t_{2}}}\left(\left(\gamma_{1}-\left(\gamma_{2}+\gamma_{3}\right) \delta-\frac{\gamma_{4}^{2}}{\alpha \gamma_{5}}\right)|\langle\bar{v}\rangle|^{2}-\frac{2 \gamma_{4}}{\alpha \gamma_{5} \delta} \Re(\langle v\rangle[\bar{v}])+\frac{1}{\alpha \gamma_{5} \delta^{2}}|[v]|^{2}\right) \mathrm{d} s \\
& \geq \int_{\Gamma_{t_{2}}}\left(\left(\gamma_{1}-\left(\gamma_{2}+\gamma_{3}\right) \delta-\frac{\gamma_{4}^{2}}{\alpha \gamma_{5}}\right)|\langle\bar{v}\rangle|^{2}-\frac{2 \gamma_{4}}{\alpha \gamma_{5} \delta}|\langle v\rangle||[v]|+\frac{1}{\alpha \gamma_{5} \delta^{2}}|[v]|^{2}\right) \mathrm{d} s \\
& =\int_{\Gamma_{t_{2}}}\left(\left(\gamma_{1}-\left(\gamma_{2}+\gamma_{3}\right) \delta-\frac{2 \gamma_{4}^{2}}{\alpha \gamma_{5}}\right)|\langle\bar{v}\rangle|^{2}+\frac{1}{\alpha \gamma_{5} \delta^{2}}\left(\gamma_{4} \sqrt{\delta}|\langle v\rangle|-|[v]|\right)^{2}\right) \mathrm{d} s .
\end{aligned}
$$

Due to the assumption (31) on $\alpha$, we obtain

$$
\Re\left(c_{1,1}(v, v)\right)-\Im\left(c_{1,1}(v, v)\right) \geq 0 .
$$

Therefore

$$
\begin{aligned}
\left|a_{1,1}(v, v)\right| & \geq \Re\left(a_{1,1}(v, v)\right)-\Im\left(a_{1,1}(v, v)\right) \\
& =\int_{\Omega_{+} \cup \Omega_{-}}\left(\frac{1}{\mu r}|\nabla(r v)|^{2}+\omega \sigma|v|^{2} r\right) \mathrm{d} r \mathrm{~d} z+\Re\left(c_{1,1}(v, v)\right)-\Im\left(c_{1,1}(v, v)\right) \\
& \geq \int_{\Omega_{+} \cup \Omega_{-}} \frac{1}{\mu r}|\nabla(r v)|^{2} \mathrm{~d} r \mathrm{~d} z \geq \frac{C}{\mu_{\text {sup }}}\|v\|_{H\left(\Omega_{-} \cup \Omega_{+}\right)}^{2} .
\end{aligned}
$$

Hence we conclude the existence and uniqueness of the solution $u^{\delta} \in H\left(\Omega-\cup \Omega_{+}\right)$using the Lax-Milgram Theorem. 


\subsection{Approximation of the impedance measurements}

In an eddy current testing with a probe of two coaxial coils (see Figure 1), each coil can be a generator and/or a receiver. We apply a current to one of the coils and we can get impedance measurements in both the two coils at each measuring position during a scan of the tube. If the coils are indexed by $k, l \in\{1,2\}$, the impedance measurement from the coil $k$ when the electromagnetic field is induced by the coil $l$ writes (see [1, (10a)], [12])

$$
\triangle Z_{k l}=\frac{2 \pi}{\mathrm{i} \omega I^{2}} \int_{\Omega_{c}^{\delta}}\left(\left(\frac{1}{\mu}-\frac{1}{\mu^{*}}\right) \frac{1}{r} \nabla\left(r u_{[k]}\right) \cdot \nabla\left(r u_{[l]}^{*}\right)-\mathrm{i} \omega\left(\sigma-\sigma^{*}\right) u_{[k]} u_{[l]}^{*} r\right) \mathrm{d} r \mathrm{~d} z,
$$

where the superscript $*$ denotes the terms in a deposit-free case, the subscripts $[k],[l]$ give the indices of the receiver coil and the generator coil respectively. In our setting $\left.\mu\right|_{\Omega_{c}^{\delta}}=\mu_{c},\left.\sigma\right|_{\Omega_{c}^{\delta}}=\sigma_{c},\left.\mu^{*}\right|_{\Omega_{c}^{\delta}}=\mu_{v}$ and $\left.\sigma^{*}\right|_{\Omega_{c}^{\delta}}=0$. In practice one usually records the impedance measurements in the two following modes [17].

$$
\left\{\begin{array}{l}
Z_{F A}=\frac{\mathrm{i}}{2}\left(\triangle Z_{11}+\triangle Z_{21}\right) \quad \text { absolute mode, } \\
Z_{F 3}=\frac{\mathrm{i}}{2}\left(\triangle Z_{11}-\triangle Z_{22}\right) \quad \text { differential mode. }
\end{array}\right.
$$

Since the permeability of copper $\mu_{c}$ is the same as the background permeability for vacuum $\mu_{v}$ (see Table 1) the impedance measurement writes

$$
\triangle Z_{k l}=-\frac{2 \pi}{I^{2}} \int_{\Omega_{c}^{\delta}} \sigma_{c} u_{[k]} u_{[l]}^{*} r \mathrm{~d} r \mathrm{~d} z
$$

The above expression in the form of a surface integration on the deposit domain $\Omega_{c}^{\delta}$ should be approximated by a line integration on the interface $\Gamma_{t_{2}}$ since we consider here asymptotic models. We use Taylor's expansions of the integrand to obtain approximations up to desired order in $\delta$.

\section{Approximation of order 0 of the impedances}

From Section 3.1 and the expression of the transmission conditions $\mathcal{Z}_{1,0}$ (17), the electric field in the thin layer $\Omega_{c}^{\delta}$ writes

$$
u(r, z)=u^{\delta}\left(r_{t_{2}}, z\right)+\mathcal{O}(\delta) \quad r_{t_{2}}<r<r_{t_{2}}+f_{\delta}(z)
$$

Then the approximation at order 0 of the impedance measurements (33) is

$$
\begin{aligned}
\triangle Z_{k l}^{0} & =-\frac{2 \pi}{I^{2}} \int_{\Gamma_{t_{2}}} \int_{r_{t_{2}}}^{r_{t_{2}}+f_{\delta}(s)} \sigma_{c} u_{[k]}^{\delta}\left(r_{t_{2}}, s\right) u_{[l]}^{*}\left(r_{t_{2}}, s\right) r \mathrm{~d} r \mathrm{~d} s \\
& =-\frac{2 \pi}{I^{2}} \int_{\Gamma_{t_{2}}} \sigma_{c} f_{\delta}(s) u_{[k]}^{\delta}\left(r_{t_{2}}, s\right) u_{[l]}^{*}\left(r_{t_{2}}, s\right) r_{t_{2}} \mathrm{~d} s
\end{aligned}
$$

\section{Approximation of order 1 of the impedances}

From Section 3.3 we write the Taylor development of $u_{1}$

$$
\begin{aligned}
& u_{1}\left(\frac{r-r_{t_{2}}}{\delta}, z\right)=\left.u_{1}^{-}\right|_{r_{t_{2}}}+\left.\frac{1}{r_{t_{2}}}\left(\frac{\mu_{c}}{\mu_{t}} \partial_{r}\left(r u_{0}^{-}\right)-u_{0}^{-}\right)\right|_{r_{t_{2}}} \frac{r-r_{t_{2}}}{\delta}-\left.\frac{i \omega \sigma_{1} \mu_{c}}{2} u_{0}^{-}\right|_{r_{t_{2}}} \frac{\left(r-r_{t_{2}}\right)^{2}}{\delta^{2}} \\
= & \left\langle u_{1}\right\rangle-\frac{1}{2}\left[u_{1}\right]+\frac{1}{r_{t_{2}}}\left(\mu_{c}\left\langle\mu^{-1} \partial_{r}\left(r u_{0}\right)\right\rangle-\frac{\mu_{c}}{2}\left[\mu^{-1} \partial_{r}\left(r u_{0}\right)\right]-\left\langle u_{0}\right\rangle\right) \frac{r-r_{t_{2}}}{\delta}-\frac{i \omega \sigma_{1} \mu_{c}}{2}\left\langle u_{0}\right\rangle \frac{\left(r-r_{t_{2}}\right)^{2}}{\delta^{2}} .
\end{aligned}
$$


So that for $r_{t_{2}}<r<r_{t_{2}}+f_{\delta}(z)$ the asymptotic solution writes

$$
\begin{aligned}
u(r, z)= & \left\langle u^{\delta}\right\rangle-\frac{1}{2}\left[u^{\delta}\right]+\frac{1}{r_{t_{2}}}\left(\mu_{c}\left\langle\mu^{-1} \partial_{r}\left(r u^{\delta}\right)\right\rangle-\frac{\mu_{c}}{2}\left[\mu^{-1} \partial_{r}\left(r u^{\delta}\right)\right]-\left\langle u^{\delta}\right\rangle\right)\left(r-r_{t_{2}}\right) \\
& -\frac{i \omega \sigma_{1} \mu_{c}}{2}\left\langle u^{\delta}\right\rangle \frac{\left(r-r_{t_{2}}\right)^{2}}{\delta}+\mathcal{O}\left(\delta^{2}\right) \\
= & \left\langle u^{\delta}\right\rangle-\frac{1}{2}\left[u^{\delta}\right]+\left(\frac{\omega^{2} \sigma_{c}^{2} \mu_{c}^{2} f_{\delta}^{3}}{6}+\mathrm{i}\left(\frac{\omega \sigma_{c} \mu_{c} f_{\delta}}{2}-\frac{\omega \sigma_{c} \mu_{c} f_{\delta}^{2}}{4 r_{t_{2}}}-\frac{\omega \sigma_{c} \mu_{c} f_{\delta}}{8 \alpha}-\frac{1}{\alpha f_{\delta}}\right)\right)\left\langle u^{\delta}\right\rangle\left(r-r_{t_{2}}\right) \\
& +\left(\frac{1}{2 \alpha f_{\delta}}-\frac{\mathrm{i}}{\alpha \omega \sigma_{c} \mu_{c} f_{\delta}^{3}}\right)\left[u^{\delta}\right]\left(r-r_{t_{2}}\right)-\mathrm{i} \frac{\omega \sigma_{c} \mu_{c}}{2}\left\langle u^{\delta}\right\rangle\left(r-r_{t_{2}}\right)^{2}+\mathcal{O}\left(\delta^{2}\right) .
\end{aligned}
$$

The last equality is due to the transmission conditions (28) and the settings of $\gamma_{i}(i=1,2, \ldots, 5)$. The electric field $u_{[l]}^{*}$ in the deposit-free configuration satisfies the following transmission conditions on $\Gamma_{t_{2}}$

$$
\left[u_{[l]}^{*}\right]=0 \quad \text { and } \quad\left[\mu^{-1} \partial_{r}\left(r u_{[l]}^{*}\right)\right]=0 .
$$

Thus one gets the Taylor expansion

$$
u_{[l]}^{*}(r, z) r=u_{[l]}^{*}\left(r_{t_{2}}, z\right) r_{t_{2}}+\partial_{r}\left(r u_{[l]}^{*}\right)\left(r_{t_{2}}, z\right)\left(r-r_{t_{2}}\right)+\mathcal{O}\left(\left(r-r_{t_{2}}\right)^{2}\right) \quad r_{t_{2}}<r<r_{t_{2}}+f_{\delta}(z) .
$$

With the above developments, we obtain the approximation at order 1 of the impedance measurements

$$
\begin{aligned}
\triangle Z_{k l}^{1}= & -\frac{2 \pi}{I^{2}} \int_{\Gamma_{t_{2}}} \sigma_{c} z\left(u_{[k]}^{\delta}, u_{[l]}^{*} ; f_{\delta}(s)\right) \mathrm{d} s \\
\text { with } z\left(u_{[k]}^{\delta}, u_{[l]}^{*} ; f_{\delta}\right):= & \left(\left\langle u_{[k]}^{\delta}\right\rangle-\frac{1}{2}\left[u_{[k]}^{\delta}\right]\right)\left(r_{t_{2}} u_{[l]}^{*} f_{\delta}+\frac{1}{2} \partial_{r}\left(r u_{[l]}^{*}\right) f_{\delta}^{2}\right) \\
& +\left\{\left(\frac{\omega^{2} \sigma_{c}^{2} \mu_{c}^{2} f_{\delta}^{5}}{12}+\mathrm{i}\left(\frac{\omega \sigma_{c} \mu_{c} f_{\delta}^{3}}{4}-\frac{\omega \sigma_{c} \mu_{c} f_{\delta}^{4}}{8 r_{t_{2}}}-\frac{\omega \sigma_{c} \mu_{c} f_{\delta}^{3}}{16 \alpha}-\frac{f_{\delta}}{2 \alpha}\right)\right)\left\langle u_{[k]}^{\delta}\right\rangle\right. \\
& \left.+\left(\frac{f_{\delta}}{4 \alpha}-\frac{\mathrm{i}}{2 \alpha \omega \sigma_{c} \mu_{c} f_{\delta}}\right)\left[u_{[k]}^{\delta}\right]\right\} r_{t_{2}} u_{[l]}^{*}
\end{aligned}
$$

\section{Thickness reconstruction via asymptotic models}

This section proposes an inversion algorithm for layer thickness reconstruction based on gradient descent of a least square cost functional. To obtain the descent direction, we first consider the derivative of the solutions of the asymptotic models with respect to a small change in layer thickness.

\subsection{Derivative of the solution with respect to a thickness increment}

Assume that $h \in L^{\infty}\left(\Gamma_{t_{2}}\right)$ is a small thickness increment of the thin layer, i.e.

$$
f_{\delta}(z) \rightarrow f_{\delta}(z)+h(z)
$$

The derivative of the solution with respect to this thickness increment satisfies a weak formulation with same sesquilinear form but with different source term.

\subsubsection{Derivative of the asymptotic model of order 0}

We denote the solution to the asymptotic model of order 0 with a thin layer of $f_{\delta}$ thickness (19) by $u^{\delta}\left(f_{\delta}\right)=$ $u^{\delta}\left(f_{\delta}\right)(r, z)$. Then the derivative of $u^{\delta}\left(f_{\delta}\right)$ due to the increment $h$, denoted by $u^{\prime}(h)$, is given by

$$
u^{\delta}\left(f_{\delta}+h\right)=u^{\delta}\left(f_{\delta}\right)+u^{\prime}(h)+o(h), \quad \lim _{h \rightarrow 0} \frac{\|o(h)\|_{H}}{\|h\|_{L^{\infty}\left(\Gamma_{t_{2}}\right)}}=0 .
$$

$u^{\delta}\left(f_{\delta}+h\right)$ solves the same variational formulation (19) with a different $\gamma_{1}$

$$
\gamma_{1}=\omega \sigma_{c}\left(f_{\delta}(z)+h(z)\right) r_{t_{2}}
$$


We develop this variational formulation $f_{\delta}+h$ at $h=0$. The terms of order zero exactly yield the variational formulation solving $u^{\delta}\left(f_{\delta}\right)$. The terms of first order with respect to $h$ imply that $u^{\prime}$ satisfies

$$
a_{1,0}\left(u^{\prime}, v\right)=\int_{\Gamma_{t_{2}}} \mathrm{i} \omega \sigma_{c} h(s) u^{\delta} \bar{v} r \mathrm{~d} s \quad \forall v \in H(\Omega),
$$

where the sesquilinear form $a_{1,0}(\cdot, \cdot)$ is defined in (20). The same argument as in Proposition 3.1 show that

Corollary 4.1. Under the same assumption as in Proposition [3.1, the variational formulation (36) has a unique solution $u^{\prime}$ in $H(\Omega)$.

\subsubsection{Derivative of the asymptotic model of order 1}

We still denote by $u^{\delta}\left(f_{\delta}\right)$ the solution to the variational formulation (29) with layer thickness $f_{\delta}(z)$, and by $u^{\prime}(h)$ its derivatives due to a thickness increment $h(z)$ which is defined as previously where $u^{\delta}\left(f_{\delta}+h\right)$ solves the same variational formulation (29) but with the coefficients

$$
\begin{array}{ll}
\gamma_{1}=\omega \sigma_{c}\left(f_{\delta}(z)+h(z)\right) r_{t_{2}}, & \gamma_{2}=\frac{\omega^{2} \sigma_{c}^{2} \mu_{c} r_{t_{2}}\left(f_{\delta}(z)+h(z)\right)^{3}}{6 \delta}, \\
\gamma_{4}=\frac{\omega \sigma_{c} \mu_{c}\left(f_{\delta}(z)+h(z)\right)^{2}}{2 \delta}, & \gamma_{5}=\frac{\omega \sigma_{c} \mu_{c}^{2}\left(f_{\delta}(z)+h(z)\right)^{3}}{r_{t_{2}} \delta^{2}} .
\end{array}
$$

Developing the variational formulation (29) with the above coefficients $\gamma_{i}(i=1, \ldots, 5)$ for $u^{\delta}\left(f_{\delta}+h\right)$ with respect to $h$ at $h=0$, the terms of order 0 give the variational formulation for $u^{\delta}\left(f_{\delta}\right)$ and vanish, while the terms of the order 1 yield

$$
a_{1,1}\left(u^{\prime}, v\right)=\int_{\Gamma_{t_{2}}} \sigma_{c} h(s) l\left(u^{\delta}, v ; f_{\delta}(s)\right) \mathrm{d} s, \quad \forall v \in H\left(\Omega_{-} \cup \Omega_{+}\right),
$$

where

$$
l\left(u^{\delta}, v ; f_{\delta}\right):=\left(\frac{3}{4} \mathrm{i} \omega r_{t_{2}}+\frac{1}{2} \omega^{2} \sigma_{c} \mu_{c} r_{t_{2}} f_{\delta}^{2}-\mathrm{i} \omega f_{\delta}\right)\left\langle u^{\delta}\right\rangle\langle\bar{v}\rangle-\frac{r_{t_{2}}}{2 \sigma_{c} \mu_{c} f_{\delta}^{2}}\left(\left\langle u^{\delta}\right\rangle[\bar{v}]+\left[u^{\delta}\right]\langle\bar{v}\rangle\right)-\frac{3 \mathrm{i} r_{t_{2}}}{\alpha \omega \sigma_{c}^{2} \mu_{c}^{2} f_{\delta}^{4}}\left[u^{\delta}\right][\bar{v}] .
$$

Corollary 4.2. Under the same assumptions as in Proposition 3.2, the variational formulation (37) has a unique solution $u^{\prime} \in H\left(\Omega_{-} \cup \Omega_{+}\right)$.

\subsection{Adjoint state and derivative of the impedance measurements}

Since the cost functional makes use of the impedance measurements as input signals, in order to apply gradient descent, one should also compute the derivative of the impedance measurement due to a small change in layer thickness $\left(f_{\delta} \rightarrow f_{\delta}+h\right)$. We denote by $\triangle Z_{k l}\left(f_{\delta}\right)$ the impedance measurement for a thin layer with $f_{\delta}(z)$ in thickness, and by $\triangle Z_{k l}^{\prime}$ its derivative with regard to $h$

$$
\triangle Z_{k l}\left(f_{\delta}+h\right)=\triangle Z_{k l}\left(f_{\delta}\right)+\triangle Z_{k l}^{\prime}+o(h) .
$$

For $\triangle Z_{k l}^{0}$, the approximation to $\triangle Z_{k l}$ of order 0 shown in (34), its derivative writes

$$
\left(\triangle Z_{k l}^{0}\right)^{\prime}=-\frac{2 \pi}{I^{2}} \int_{\Gamma_{t_{2}}} \sigma_{c}\left(h(s) u_{[k]}^{\delta} u_{[l]}^{*} r_{t_{2}}+f_{\delta}(s) u_{[k]}^{\prime} u_{[l]}^{*} r_{t_{2}}\right) \mathrm{d} s .
$$

Similarly, the approximation $\triangle Z_{k l}^{1}$ to $\triangle Z_{k l}$ of order 1 given by (35) has the derivative

$$
\left(\triangle Z_{k l}^{1}\right)^{\prime}=-\frac{2 \pi}{I^{2}} \int_{\Gamma_{t_{2}}} \sigma_{c}\left(z\left(u_{[k]}^{\prime}, u_{[l]}^{*} ; f_{\delta}(s)\right)+\left(\partial_{f} z\right)\left(u_{[k]}^{\delta}, u_{[l]}^{*} ; f_{\delta}(s)\right) h(s)\right) \mathrm{d} s,
$$

with $\left(\partial_{f} z\right)\left(u_{[k]}^{\delta}, u_{[l]}^{*} ; f_{\delta}\right):=\left(\left\langle u_{[k]}^{\delta}\right\rangle-\frac{1}{2}\left[u_{[k]}^{\delta}\right]\right)\left(r_{t_{2}} u_{[l]}^{*}+\partial_{r}\left(r u_{[l]}^{*}\right) f_{\delta}\right)$

$$
\begin{aligned}
& +\left\{\left(\frac{5 \omega^{2} \sigma_{c}^{2} \mu_{c}^{2} f_{\delta}^{4}}{12}+\mathrm{i}\left(\frac{3 \omega \sigma_{c} \mu_{c} f_{\delta}^{2}}{4}-\frac{\omega \sigma_{c} \mu_{c} f_{\delta}^{3}}{2 r_{t_{2}}}-\frac{3 \omega \sigma_{c} \mu_{c} f_{\delta}^{2}}{16 \alpha}-\frac{1}{2 \alpha}\right)\right)\left\langle u_{[k]}^{\delta}\right\rangle\right. \\
& \left.+\left(\frac{1}{4 \alpha}+\frac{\mathrm{i}}{2 \alpha \omega \sigma_{c} \mu_{c} f_{\delta}^{2}}\right)\left[u_{[k]}^{\delta}\right]\right\} r_{t_{2}} u_{[l]}^{*}
\end{aligned}
$$


We remark that the above derivatives (39) and (40) are implicit expressions with respect to the thickness increment $h(z)$, as in their expressions appears the derivative of the solution $u_{[k]}^{\prime}(h)$ which solves either (36) or (37) involving $h$ in the second member. In the following we take the approach of Hadamard's representation, that is to introduce an adjoint state such that the derivatives of such impedance measurements will get rid of the terms $u_{[k]}^{\prime}$ and only depend on $h$ explicitly.

\section{Asymptotic model of order 0}

To write $\left(\triangle Z_{k l}^{0}\right)^{\prime}$ explicitly on the thickness increment $h$ (independent of $u_{[k]}^{\prime}$ ), we introduce the adjoint state $p_{[l]}$ satisfying

$$
a_{1,0}^{*}\left(p_{[l]}, v\right)=-\int_{\Gamma_{t_{2}}} \mathrm{i} \omega \sigma_{c} f_{\delta}(s) \overline{u_{[l]}^{*}} \bar{v} r_{t_{2}} \mathrm{~d} s \quad \forall v \in H(\Omega)
$$

where

$$
a_{1,0}^{*}(p, v):=\overline{a_{1,0}(v, p)} \quad \forall(p, v) \in H(\Omega)^{2} .
$$

Proposition 4.3. Under the same assumptions as in Proposition [3.1, the variational formulation of the adjoint problem (41) has a unique solution $p_{[l]}$ in $H(\Omega)$. If $u_{[k]}^{\delta}$ is the solution to the asymptotic model using $\mathcal{Z}_{1,0}$ transmission conditions (19), then the derivative of the impedance measurement $\triangle Z_{k l}$ due to a thickness increment $h(z)$ writes

$$
\left(\triangle Z_{k l}^{0}\right)^{\prime}=-\frac{2 \pi}{I^{2}} \int_{\Gamma_{t_{2}}} \sigma_{c} h(s) u_{[k]}^{\delta}\left(u_{[l]}^{*}+\overline{p_{[l]}}\right) r_{t_{2}} \mathrm{~d} s .
$$

Proof. One obtains with the same argument as in Proposition 3.1 the existence and uniqueness of solution to the adjoint problem (41). From (41) and (36), we have

$$
\int_{\Gamma_{t_{2}}} \mathrm{i} \omega \sigma_{c} f_{\delta}(s) u_{[k]}^{\prime} u_{[l]}^{*} r_{t_{2}} \mathrm{~d} s=\overline{a_{1,1}^{*}\left(p_{[l]}, u_{[k]}^{\prime}\right)}=a_{1,0}\left(u_{[k]}^{\prime}, p_{[l]}\right)=\int_{\Gamma_{t_{2}}} \mathrm{i} \omega \sigma_{c} h(s) u_{[k]}^{\delta} \overline{p_{[l]}} r_{t_{2}} \mathrm{~d} s
$$

which, together with (39), implies (43).

\subsubsection{Asymptotic model of order 1}

We look for an adjoint state such that the derivative of the impedance measurement $\left(\triangle Z_{k l}^{1}\right)^{\prime}$ has an explicit expression on the thickness increment $h(z)$ independent of the derivative $u_{[k]}^{\prime}$ of the solution. The adjoint state $p_{[l]} \in H\left(\Omega_{-} \cup \Omega_{+}\right)$is the unique solution of the following variational problem

$$
\begin{aligned}
a_{1,1}^{*}\left(p_{[l]}, v\right) & =\int_{\Gamma_{t_{2}}} \sigma_{c} \overline{z\left(v, u_{[l]}^{*} ; f_{\delta}(s)\right)} \mathrm{d} s \quad \forall v \in H\left(\Omega_{-} \cup \Omega_{+}\right), \\
\text {where } \quad a_{1,1}^{*}\left(p_{[l]}, v\right) & :=\overline{a_{1,1}\left(v, p_{[l]}\right)} .
\end{aligned}
$$

Proposition 4.4. Under the same assumptions as in Proposition 3.2, the adjoint problem (44) has a unique solution $p_{[l]} \in H\left(\Omega_{-} \cup \Omega_{+}\right)$. If $u_{[k]}^{\delta}$ is the solution to the asymptotic model using $\mathcal{Z}_{1,1}$ transmission conditions (29), then the derivative of the impedance measurement $\triangle Z_{k l}$ due to a thickness increment $h(z)$ writes

$$
\left(\triangle Z_{k l}^{1}\right)^{\prime}=-\frac{2 \pi}{I^{2}} \int_{\Gamma_{t_{2}}} \sigma_{c} h(s)\left(l\left(u_{[k]}^{\delta}, p_{[l]} ; f_{\delta}(s)\right)+\left(\partial_{f} z\right)\left(u_{[k]}^{\delta}, u_{[l]}^{*} ; f_{\delta}(s)\right)\right) \mathrm{d} s
$$

where the form $l\left(\cdot, \cdot ; f_{\delta}\right)$ is given by (38).

Proof. The existence of uniqueness of solution $p_{[l]}$ to the variational problem (44) can be concluded from the same argument in the proof of Proposition 3.2. From (37) and (44), we have

$$
\int_{\Gamma_{t_{2}}} \sigma_{c} z\left(u_{[k]}^{\prime}, u_{[l]}^{*} ; f_{\delta}(s)\right) \mathrm{d} s=\overline{a_{1,1}^{*}\left(p_{[l]}, u_{[k]}^{\prime}\right)}=a_{1,1}\left(u_{[k]}^{\prime}, p_{[l]}\right)=\int_{\Gamma_{t_{2}}} \sigma_{c} h(s) l\left(u_{[k]}^{\delta}, p_{[l]} ; f_{\delta}(s)\right) \mathrm{d} s .
$$

The above equality and (40) yield (45). 


\subsection{Thickness reconstruction by minimizing a least square cost functional}

Recall that we use impedance signals measured during a eddy current probe scan along the tube axis covering a section from $z_{\min }$ to $z_{\max }$ to reconstruct the layer thickness. On each probe position $\zeta \in\left[z_{\min }, z_{\max }\right]$ an impedance signal is measured and denoted by $Z_{\text {meas }}(\zeta)$, which can be either in FA mode or in F3 mode (see (32) $)$. We apply the gradient descent algorithm which minimizes a least square cost functional of the layer thickness $f_{\delta}(z)$ given by

$$
\mathcal{J}\left(f_{\delta}\right):=\int_{z_{\min }}^{z_{\max }}\left|Z\left(f_{\delta} ; \zeta\right)-Z_{\text {meas }}(\zeta)\right|^{2} \mathrm{~d} \zeta
$$

where $Z\left(f_{\delta} ; \zeta\right)$ is the numerical approximation of impedance measurement in the corresponding FA mode or in F3 mode at probe position $\zeta$ with the simulated thin layer of thickness $f_{\delta}$. Hence, the derivative of the cost functional due to a small increment $h$ of the layer thickness $f_{\delta}$ writes

$$
\begin{aligned}
& \mathcal{J}^{\prime}(h)=\int_{z_{\min }}^{z_{\max }} 2 \Re\left(Z^{\prime}(h ; \zeta)\left(\overline{Z\left(f_{\delta} ; \zeta\right)-Z_{\text {meas }}(\zeta)}\right)\right) \mathrm{d} \zeta \\
& \text { where } \\
& Z^{\prime}(h)= \begin{cases}Z_{F A}^{\prime}=\frac{\mathrm{i}}{2}\left(\triangle Z_{11}^{\prime}+\triangle Z_{21}^{\prime}\right) & \text { FA mode, } \\
Z_{F 3}^{\prime}=\frac{\mathrm{i}}{2}\left(\triangle Z_{11}^{\prime}-\triangle Z_{22}^{\prime}\right) & \text { F3 mode. }\end{cases}
\end{aligned}
$$

In the following, whether the asymptotic model and the impedance approximation are of order 0 or 1 , we always use the same notation $\mathcal{J}, \mathcal{J}^{\prime}$ for the cost functional or its derivative. We have

$$
\begin{gathered}
\mathcal{J}^{\prime}(h)=-\frac{\pi}{I^{2}} \int_{\Gamma_{t_{2}}} \sigma_{c} h(s) g(s) \mathrm{d} s, \\
\text { where } \quad g= \begin{cases}g_{11}+g_{21} & \text { FA mode, } \\
g_{11}-g_{22} & \text { F3 mode. }\end{cases}
\end{gathered}
$$

The functions $g_{k l}(k, l=1,2)$ are defined according to the expression of the derivative of the impedance measurements involving adjoint states. If we consider both asymptotic model and approximation of impedance measurements of order 0 , the expression of $\left(\triangle Z_{k l}^{0}\right)^{\prime}$ given by (43) yields

$$
g_{k l}:=\int_{z_{\min }}^{z_{\max }} \Re\left(\mathrm{i} u_{[k]}^{\delta}\left(u_{[l]}^{*}+\overline{p_{[l]}}\right) r_{t_{2}}\left(\overline{Z\left(f_{\delta} ; \zeta\right)-Z_{\text {meas }}(\zeta)}\right)\right) \mathrm{d} \zeta,
$$

while for the case of approximation order 1 , the expression of $\left(\triangle Z_{k l}^{1}\right)^{\prime}$ given by (45) implies

$$
g_{k l}:=\int_{z_{\min }}^{z_{\max }} \Re\left(\mathrm{i}\left(l\left(u^{\delta}, p_{[l]} ; f_{\delta}\right)+\left(\partial_{f} z\right)\left(u_{[k]}^{\delta}, u_{[l]}^{*} ; f_{\delta}\right)\right)\left(\overline{Z\left(f_{\delta} ; \zeta\right)-Z_{\text {meas }}(\zeta)}\right)\right) \mathrm{d} \zeta .
$$

We observe that $h=g$ is a descent direction that decreases the cost functional

$$
\mathcal{J}^{\prime}(g)=-\frac{\pi}{I^{2}} \int_{\Gamma_{t_{2}}} \sigma_{c}|g(s)|^{2} \mathrm{~d} s \leq 0
$$

Therefore, we propose an iterative inversion algorithm to reconstruct the thin layer thickness as follows. We set $\epsilon>0$ a threshold for the stopping rule.

- Initialize with a guess of the thin layer thickness $f_{\delta}(z)$.

- Step $k$ :

- Evaluate the numerical approximation of the impedance measurements $Z\left(f_{\delta}, \zeta\right)\left(\zeta \in\left[z_{\min }, z_{\max }\right]\right)$ (34) or (35) with the actual layer thickness $f_{\delta}(z)$. If the following criteria is satisfied

$$
\mathcal{J}\left(f_{\delta}\right)<\epsilon \int_{z_{\min }}^{z_{\max }}\left|Z_{\text {meas }}(\zeta)\right|^{2} \mathrm{~d} \zeta
$$


then $f_{\delta}$ is the reconstructed thickness and we stop the algorithm. Otherwise we solve the direct problem (19) or (29) and adjoint problem (41) or (44) with $f_{\delta}$ and get a thickness increment $h=t g$ where $g=g_{11}+g_{21}$ or $g=g_{11}-g_{22}$ according to the measurement mode with $g_{k l}(k, l=1,2)$ given by (48) or (49) and where $t>0$ is a sufficiently small threshold. We choose $t$ such that $\|h\|_{\infty}=5 \mu m$ in the first two loops and then it remains fixed in the following loops.

- Update the layer thickness $f_{\delta} \rightarrow f_{\delta}+h$ and go to step $k+1$.

\section{$5 \quad$ Numerical tests}

\subsection{Numerical validation of the 2-D asymptotic models}

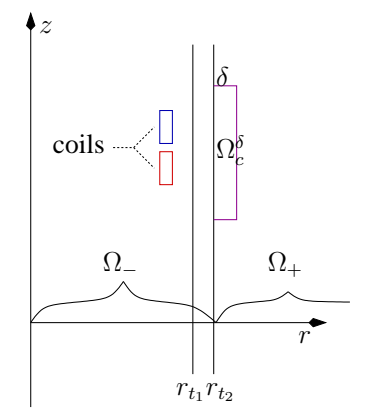

Figure 2: Sketch of a 2-D axisymmetrical full model with a thin layer $\Omega_{c}^{\delta}$ with constant thickness $\delta$. The thickness is exaggerated in the sketch.

We test the asymptotic models in their variational formulations using $\mathcal{Z}_{1, n}(n=0,1)$ transmission conditions given by the previous sections. The general geometrical configurations shown in Figure 1 or Figure 2 are as follows. The two coils involved are represented by two rectangles with $0.67 \mathrm{~mm}$ in length (radial direction) and $2 \mathrm{~mm}$ in height (longitudinal direction). They are located $7.83 \mathrm{~mm}$ away from the $z$-axis and have a distance of $0.5 \mathrm{~mm}$ between them. The SG tube measures $9.84 \mathrm{~mm}$ in radius for the interior interface and $11.11 \mathrm{~mm}$ for the exterior interface. The permeability of tube is $\mu_{t}=1.01 \mu_{v}$, and its conductivity is $\sigma_{t}=9.7 \times 10^{5} \mathrm{~S} / \mathrm{m}$.

We first verify that the transmission conditions $\mathcal{Z}_{1, n}(n=0,1)$ yield the right order of approximation. Different from the 1-D case where one has analytical solution of the full model (see [9]), the 2-D full model does not yield explicit analytical solution. Hence only numerical approximations of the full model can serve as comparison references. To ensure the accuracy of these numerical approximations which would deteriorate as the deposit layer has tiny thickness and high conductivity, we rather consider layers with larger thicknesses and lower conductivities. We fix the rescaled deposit conductivity $\sigma_{1}=10^{3} \mathrm{~S} / \mathrm{m}$ for a range of layer thickness $\delta$ from $10^{-3} \mathrm{~m}$ to $10^{-2.5} \mathrm{~m}$. We recall that under these settings, the effective deposit conductivity $\sigma_{c}=\sigma_{1} / \delta$ varies from $10^{5.5} \mathrm{~S} / \mathrm{m}$ to $10^{6} \mathrm{~S} / \mathrm{m}$. The deposit covers the shell side of the tube homogeneously over a section of $20 \mathrm{~mm}$ in height (see Figure2).

For the full model, we compute the solution numerically with the software FreeFEM ++ (c.f. [11]) on a mesh that is adaptively refined with respect to this solution with a maximum edge size $h_{\max }=1.25 \mathrm{~mm}$ as well as P1 finite elements on the computational domain $B_{r_{*}, z_{*}}=\left\{(r, z): 0 \leq r \leq r_{*},-z_{*} \leq z \leq z_{*}\right\}$ with $r_{*}=30 \mathrm{~mm}$ and $z_{*}=41 \mathrm{~mm}$. Then we build the asymptotic models using either $\mathcal{Z}_{1,0}$ (see (17)) or $\mathcal{Z}_{1,1}$ (see (28) and we take $\alpha=2 / 3$ ) transmission conditions on $\Gamma_{t_{2}}$. The mesh is adaptively refined with respect to the solutions with a maximum edge size $h_{\max }=2.5 \mathrm{~mm}$ - which is two times larger than the edge size used in the full model - and P1 finite elements.

We shall give the error estimate in the $L_{1 / 2}^{2}$-norm. The weighted function space $L_{1 / 2}^{2}(\Omega)$ for $\Omega \subset \mathbb{R}_{+}^{2}$ is defined by $L_{1 / 2}^{2}(\Omega):=\left\{v(r, z): r^{1 / 2} v(r, z) \in L^{2}(\Omega)\right\}$.

Figure 3 shows the relative differences in $L_{1 / 2}^{2}$ norm between solutions on $\Omega_{-}$(i.e. the electric field inside the tube and in the tube wall) from asymptotic models using $\mathcal{Z}_{1, n}, n=0,1$ and that of the full model. The slope for $n=0$ is about 0.8 , while the slope for $n=1$ is about 1.6 . 


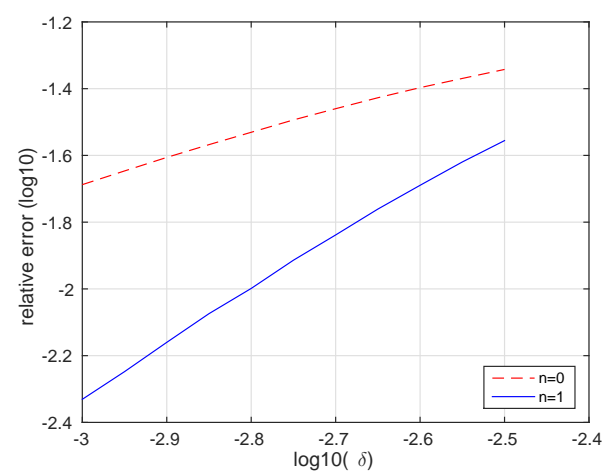

Figure 3: Relative error of the asymptotic models using $\mathcal{Z}_{1, n}$ transmission conditions, $n=0,1$. Synthetic deposit conductivities and layer thicknesses.

We then consider a thin layer of copper, with permeability $\mu_{c}=\mu_{v}$ and conductivity $\sigma_{c}=5.8 \times 10^{6} \mathrm{~S} / \mathrm{m}$. It covers the shell side of the tube with $10 \mathrm{~mm}$ in height. The thickness of the thin layer $f_{\delta}(z)=\delta$ is constant and takes value in the range from $10 \mu \mathrm{m}$ to $200 \mu \mathrm{m}$.

To have a good simulation of the thin deposit layer in the full model, at least 4 layers of mesh elements are used in the thickness direction. That makes the degrees of freedom of the finite element space exceed 11000 for the computational domain $B_{r_{*}, z_{*}}$. To ensure that this full model has enough accuracy, we refine again the mesh and observe that there is no significant difference appears. For the asymptotic models, we use the same meshing techniques as above for the synthetic example. The degrees of freedom of the finite element space are about 4000 on $B_{r_{*}, z_{*}}$.

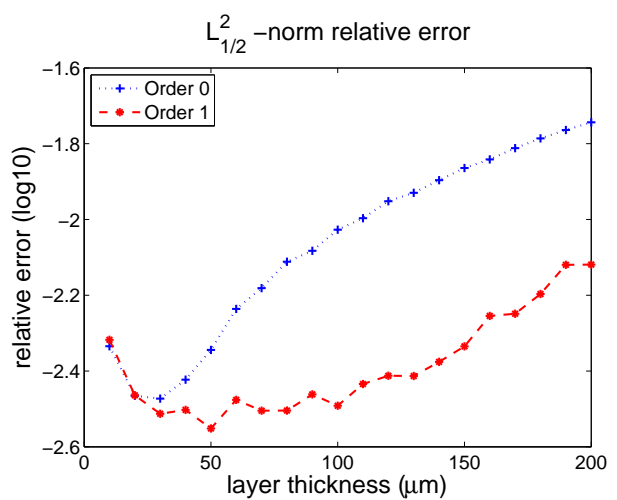

Figure 4: Relative error of the asymptotic models using $\mathcal{Z}_{1, n}$ transmission conditions, $n=0,1$. Copper layer.

We compare in Figure 4 the relative errors of the asymptotic models with regard to the full model in $L_{1 / 2}^{2}\left(B_{r_{*}, z_{*}}\right)$-norm. One observes that the asymptotic model using $\mathcal{Z}_{1,0}$ gives already a good approximation of the full model with a relative error less than $1 \%$ for $f_{\delta}(z)<100 \mu m$. But if $f_{\delta}(z)$ increases over $100 \mu m$, its precision deteriorates. The asymptotic model using $\mathcal{Z}_{1,1}$ conditions is always a good approximation of the full model for the layer thickness $f_{\delta}(z)$ under $200 \mu m$.

\subsection{Numerical approximation of impedance measurements}

We consider the same setting with copper deposit layer as in Section 5.1 for the full model (reference) and for the asymptotic models using $\mathcal{Z}_{1,0}$ and $\mathcal{Z}_{1,1}$ transmission conditions. The eddy current probe is located at the center position in the vertical direction with regard to the thin layer of deposit in copper. We compare the impedance measurement signals in FA mode (see (32)) at this position between the full model and the asymptotic models.

Figure 5a shows the impedance measurements in their real and imaginary part. One observes that the signals given by the asymptotic model using $\mathcal{Z}_{1,1}$ transmission conditions are closer to those from the full 


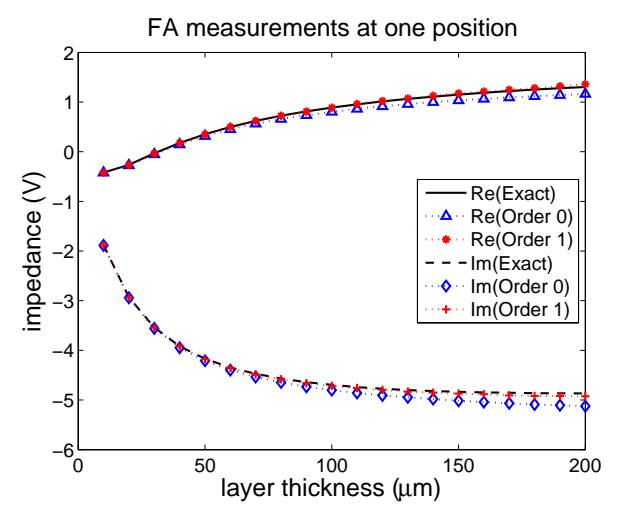

(a) one position FA measurements

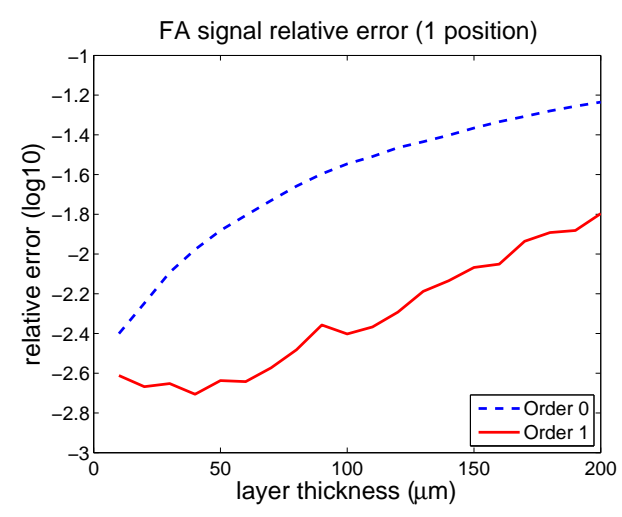

(b) relative errors

Figure 5: Approximation of impedance measurements using asymptotic models with $\mathcal{Z}_{1, n}, n=0,1$.

model than the signals obtained from the asymptotic model using $\mathcal{Z}_{1,0}$ conditions. We confirm this observation by Figure $5 \mathrm{~b}$ which illustrates the relative error of the signals in FA mode. The asymptotic model using $\mathcal{Z}_{1,0}$ transmission conditions gives a good approximation only for small layer thickness (under $40 \mu \mathrm{m}$ ), while the asymptotic model using $\mathcal{Z}_{1,1}$ yields an accurate simulation for a large range of layer thickness interested for instance, the relative error in impedance measurements is under $1 \%$ if the thickness is less than $150 \mu m$.

\subsection{Numerical tests of thickness reconstruction}

In this section, we consider some numerical examples of thickness reconstruction of copper layers. The signals of impedance measurements used for inversion are obtained from a full model. Its numerical settings are the same for the reference full model in Section 5.1. In the inversion algorithm, we use asymptotic models to resolve forward problems. The mesh methods for the asymptotic models are the same as those for asymptotic models in Section 5.1. We recall the stopping rule

$$
\mathcal{J}\left(f_{\delta}\right) \leq \epsilon \int_{z_{\min }}^{z_{\max }}\left|Z_{\text {meas }}(\zeta)\right|^{2} \mathrm{~d} \zeta,
$$

where $\epsilon$ is a chosen threshold. We take $\epsilon=10^{-4}$ such that the relative error of the impedance measurements obtained with the reconstructed thin layer is under $1 \%$ of the real measurements.

\subsubsection{Parametrized thin layers}

We consider an axisymmetric thin layer covering vertically a section of $10 \mathrm{~mm}$ of the tube's shell side. We assume that the layer thickness $f_{\delta}(z)$ is constant over this section and vanishes elsewhere. Thus in the 2-D representation with $(r, z)$ coordinates, the thin layer is a rectangle with $f_{\delta}$ in $r$-direction and $10 \mathrm{~mm}$ in $z$ direction. Since there is only one parameters to reconstruct, we need only the impedance signal in FA mode at one measuring position.

\begin{tabular}{lrrrrr}
\hline target thickness $(\mu m)$ & 10 & 20 & 30 & 50 & 75 \\
\hline reconstruction $\mathcal{Z}_{1,0}$ & 9.86 & 19.61 & 29.34 & N.A. & N.A. \\
reconstruction $\mathcal{Z}_{1,1}$ & 9.89 & 19.69 & 29.41 & 48.30 & 71.03 \\
\hline
\end{tabular}

Table 2: Reconstruction layer thickness using FA signals.

Table 2 gives the reconstruction results with the asymptotic models using either $\mathcal{Z}_{1,0}$ or $\mathcal{Z}_{1,1}$ transmission conditions. We observes that for a small target thickness (say, less than $30 \mu m$ ), both models yield satisfying reconstruction results. However, when the target thickness gets larger, the inversion algorithm with the asymptotic model using $\mathcal{Z}_{1,0}$ conditions does not converge. In fact, due to the modeling error, the minimum of the cost functional is bounded away from 0 . For instance, for a target thickness $50 \mu m$, Figure 6 shows the 
relative cost functional obtained with the asymptotic model using $\mathcal{Z}_{1,0}$ transmission conditions. Its minimum is about $10^{-2.5}$, still far away from $10^{-4}$ which is our chosen stopping threshold for the inversion algorithm.

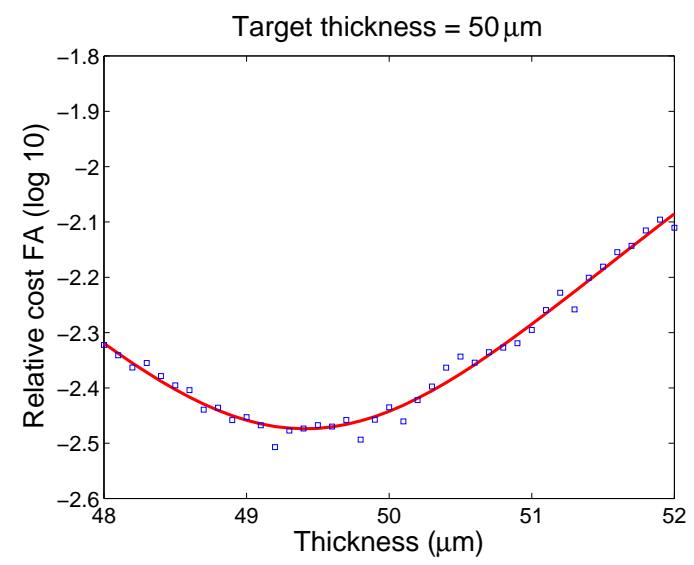

Figure 6: Cost functional for asymptotic model using $\mathcal{Z}_{1,0}$ around the target thickness.

\subsubsection{Reconstruction of arbitrary thin layers}

We consider some arbitrary thin layers of copper with variable thickness covering the tube's shell side with different height $(10 \mathrm{~mm}$ for the case in Figure $7 \mathrm{a}$ and $15 \mathrm{~mm}$ for that in Figure $7 \mathrm{~b}$ ) . In the inversion algorithm, we use the asymptotic model with $\mathcal{Z}_{1,1}$ transmission conditions on a section of exterior tube wall $\Gamma_{t_{2}}$ of height $20 \mathrm{~mm}$ (Figure 7a) or $30 \mathrm{~mm}$ (Figure 7b) and we initialize the inversion algorithm with zero layer thickness over all this concerned section. We remark that this initialization setting is reasonable since the location of copper layer on the tube is roughly indicated by the experimental eddy current signal and is included in the interested section in the inversion algorithm. During the iterative reconstruction, the layer thickness $f_{\delta}(z)$ is assumed to be a piecewise linear function on an equidistant grid over the interested section, with $0.5 \mathrm{~mm}$ between two neighboring points of the grid. Therefore, we reconstruct in fact a finite number of values of $f_{\delta}$ on the grid points. Figures 7 show some reconstruction results using either FA signals or F3 signals. In the first example (Figure 7a), we take the signals from 41 probe positions with $0.5 \mathrm{~mm}$ between each two neighboring positions. In the second example (Figure $7 \mathrm{~b}$ ), signals from 61 probe positions are used for reconstruction. Both examples show satisfying reconstruction of the thin layers.

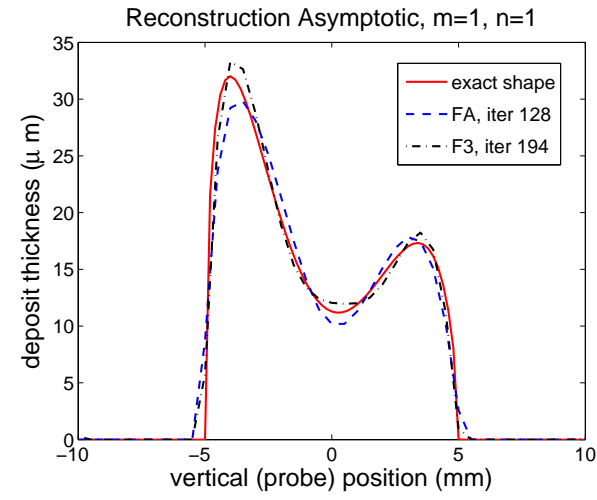

(a)

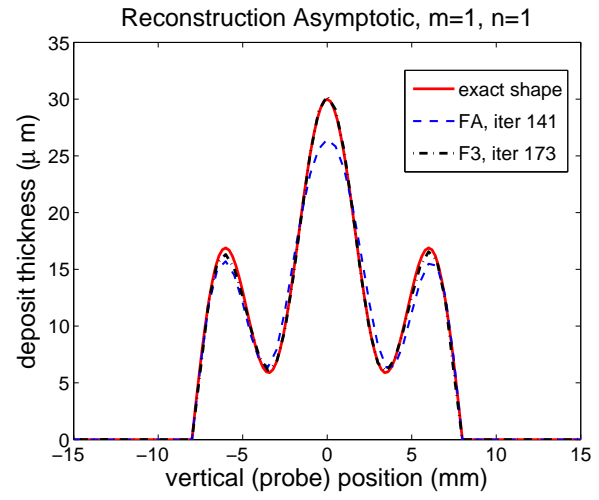

(b)

Figure 7: Reconstruction of some arbitrary thin layers.

Finally we test the robustness of reconstruction algorithm subject to noisy impedance signals. We add an artificial noise to the input impedance signals. Precisely, for each probe position the noisy impedance measurement is a uniformly distributed random complex number on a circle centered at the original impedance measurement and with radius of the noise level times the magnitude of the original impedance measurement. 
In our tests, we choose the noise level as $5 \%$ or $10 \%$. Obviously the parameter $\epsilon$ in the stopping rule should be adapted to the noise level such that the inversion algorithm converges. We set $\epsilon=(1 \%+\text { noise level })^{2}$, that is $\epsilon=0.0036$ for input signals with $5 \%$ noise level, or $\epsilon=0.0121$ for input signals with $10 \%$ noise level. Figure 8 shows the reconstruction results from these noisy impedance measurements with the corresponding parameter $\epsilon$ in the stopping rule of the inversion algorithm. We conclude that reconstructions using impedance measurements of F3 mode give more satisfactory results when the signals are noised.

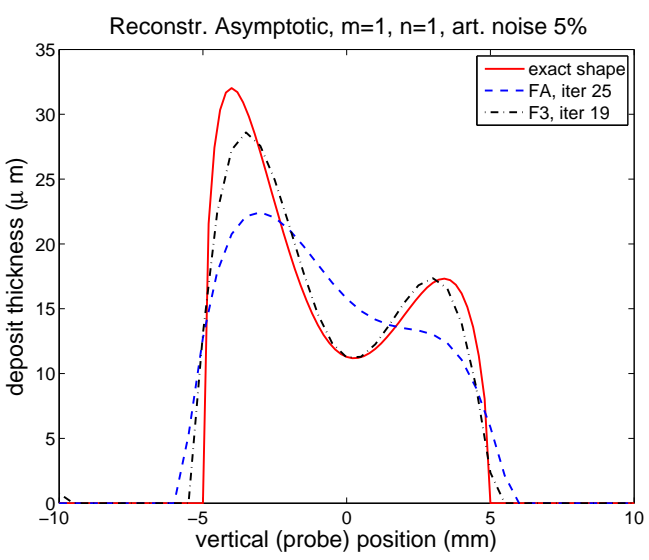

(a) artificial noise level $5 \%$

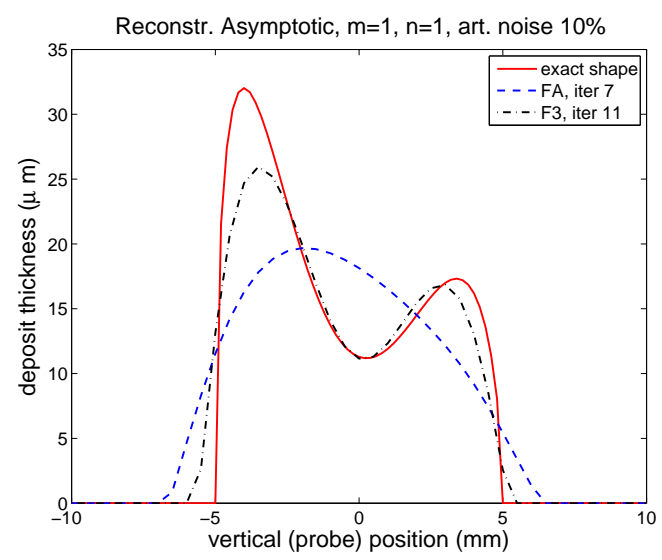

(b) artificial noise level $10 \%$

Figure 8: Reconstruction of some arbitrary thin layers from noisy impedance signals.

\section{References}

[1] B. A. Auld and J. C. Moulder. Review of advances in quantitative eddy current nondestructive evaluation. Journal of Nondestructive Evaluation, 18:3-36, 1999. 10.1023/A:1021898520626.

[2] Xavier Claeys. Analyse asymptotique et numérique de la diffraction d'ondes par des fils minces. PhD thesis, Université de Versailles-Saint Quentin en Yvelines, 122008.

[3] Xavier Claeys and F. Collino. Asymptotic and numerical analysis for holland and simpson's thin wire formalism. J. Computational Applied Mathematics, 235(15):4418-4438, 2011.

[4] Bérangère Delourme. Modèles et asymptotiques des interfaces fines et périodiques. PhD thesis, Université Pierre et Marie Curie.

[5] Bérangère Delourme and Xavier Claeys. High order asymptotics for wave propagation across thin periodic interfaces. March 2012.

[6] Bérangère Delourme, Houssem Haddar, and Patrick Joly. Approximate Models for Wave Propagation Across Thin Periodic Interfaces. Jounal de mathématiques pures et appliquées, 98(1):28-71, 2012.

[7] Pascal Jean Frey and Paul-Louis George. Mesh generation. ISTE, London; John Wiley \& Sons, Inc., Hoboken, NJ, second edition, 2008. Application to finite elements.

[8] Bojan B. Guzina and Marc Bonnet. Small-inclusion asymptotic of misfit functionals for inverse problems in acoustics. Inverse Problems, 22(5):1761-1785, 2006.

[9] Houssem Haddar and Zixian Jiang. Validity of some asymptotic models for eddy current inspection of highly conducting thin deposits. Rapport de recherche RR-8556, INRIA, July 2014.

[10] Houssem Haddar, Zixian Jiang, and Armin Lechleiter. Artificial boundary conditions for axisymmetric eddy current probe problems. Computers $8 \mathcal{G}$ Mathematics with Applications, 68(12, Part A):1844 - 1870 , 2014 . 
[11] Frédéric Hecht. New development in Freefem++. J. Numer. Math., 20(3-4):251-265, 2012.

[12] Zixian Jiang, Houssem Haddar, Armin Lechleiter, and Mabrouka El-Guedri. Identification of deposits in steam generators via shape optimization techniques. submitted.

[13] Patrick Joly and Sébastien Tordeux. Matching of asymptotic expansions for wave propagation in media with thin slots. I. The asymptotic expansion. Multiscale Model. Simul., 5(1):304-336 (electronic), 2006.

[14] Tordeux Síębastien Joly, Patrick. Matching of asymptotic expansions for waves propagation in media with thin slots ii: The error estimates. ESAIM: Mathematical Modelling and Numerical Analysis, 42(2):193221,32008 .

[15] Ozgur Ozdemir, Houssem Haddar, and Ali Yaka. Reconstruction of the electromagnetic field in layered media using the concept of approximate transmission conditions. Antennas and Propagation, IEEE Transactions on, 59(8):2964-2972, 2011.

[16] Won-Kwang Park. Inverse Scattering from Two-Dimensional Thin Inclusions and Cracks. PhD thesis, École Polytechnique.

[17] Grégoire Pichenot, Denis Prémel, Thierry Sollier, and V Maillot. Development of a 3d electromagnetic model for eddy current tubing inspection: Application to steam generator tubing. In AIP Conference Proceedings, volume 700, pages 321-328, 2003. 10.1063/1.1711640.

[18] Clair Poignard. Approximate transmission conditions through a weakly oscillating thin layer. Mathematical Methods in the Applied Sciences, 32(4):435-453, 2009.

[19] Kersten Schmidt. High-order numerical modelling of highly conductive thin sheets. PhD thesis, ETH Zurich, 2008.

[20] Kersten Schmidt and Alexey Chernov. A unified analysis of transmission conditions for thin conducting sheets in the time-harmonic eddy current model. SIAM J. Appl. Math., 73(6):1980-2003, 2013.

[21] Kersten Schmidt and Sébastien Tordeux. Asymptotic expansion of highly conductive thin sheets. PAMM - Proceedings in applied mathematics and mechanics , 7:2040011-2040012, July 2008.

[22] Sébastien Tordeux. Méthodes Asymptotiques pour la Propagation des Ondes dans les Milieux comportant des Fentes. PhD thesis, Université de Versailles-Saint Quentin en Yvelines, 2004. 